\title{
Steuerungsethik lauteren Wettbewerbs und Protestantismus - Ein Beitrag zur ideengeschichtlichen Diskussion
}

Harald Herrmann

Received 9 January 2017; Accepted 19 September 2017

Zusammenfassung: Im Einklang mit jüngsten Fortschritten der Smith- und Lutherforschung wird die Protestantismusthese zum Ursprung des wettbewerblichen Kapitalismus bestätigt, aber dahingehend modifiziert, dass es sich um einen Paradigmenwechsel des lutherischen Individualismus handelt. Dafür wird hier auf das neue Menschenbild kontingenter Glaubensrechtfertigung bei Luther abgestellt sowie an die Naturrechtslehre des Thomasius bis zu Adam Smith und Kant angeknüpft. Im Hinblick auf diese Wirkungsgeschichte des individualistischen Paradigmas ergeben sich europäische Wurzeln einer wettbewerblichen Verantwortungs- und Steuerungsethik, die nicht auf den Markterfolg oder Misserfolg abstellt, sondern auf Verantwortungsbereitschaft im Sinne kompetenter Risikoidentifikation und -verarbeitung. Danach ist sittenwidriges Wettbewerbshandeln v.a. dann anzunehmen, wenn Marktrisiken ohne nachvollziehbare Gründe der Risikokontrolle auf Marktpartner oder Dritte abgewälzt werden. Auch zu zahlreichen Auslegungsproblemen zu weiteren rechtsethischen Generalklauseln des Bank-, Gesellschafts- und Wirtschaftsrechts ergeben sich neuartige Lösungsansätze.

Abstract: Based on actual advancements of historical research on Smith and Luther, the thesis of protestantism concerning the roots of competitive capitalism is being confirmed, but modified by aspects of paradigmatic revolution to individualistic anthropology. The present paper analyses the Lutheran theology of justification as a special kind of contingency doctrine and shows historical developments from Luther to Thomasius, Smith and Kant. As to the history of paradigma effects of individualism, European origins of competition ethics of responsibility can be shown, which do not refer to market performance or imperformance, but to accountability in sense of risk identification and risk control. Unfair competitive actions, therefore, are to be legally interdicted as unethical competition behaviour when market risks are transferred to market partners or to third persons without good reasons of risk control. Some further conclusions can be made to ethical rules of banking and companies' law as well as to the general law of commerce.

(C) De Gruyter Open Sp. z 0.0.

\section{Das Humanitätsanliegen}

Nach den Finanzkrisen ist der bis dahin vorherrschende Konsens über die Vorzüge der Marktwirtschaft beträchtlich in Verruf geraten, obgleich dieser v.a. nach dem Mauerfall stark an Überrzeugungskraft gewonnen hat (Luce 2017, S. 29 mit weiteren Nachweisen). Wo nicht die Kluft von reich und arm ganz generell beklagt oder Re-Regulierung verlangt wird (Schmidt 2009) ${ }^{1}$, da mehren sich doch auch zurückhaltende Stimmen

1 Vgl. zur grundlegenden Kritik am Staatsversagen im sogenannten Thatcherism Jann und Wegrich (2004), S. 198; Sturm (2009), S. 18 f.; zu entsprechenden Problemen globaler Wirtschaftspolitiken vgl. Teubner (2012), S. 11 ff.; z.T. überzogen allerdings die Kritik an ordoliberalen Wirtschaftsverfassungskonzepten Ebd., S. 56 ff. 
für ein Mehr an Menschlichkeit und Mit-Verantwortung im wettbewerblichen Marktgeschehen ${ }^{2}$ und für die Stärkung kultureller Grundlagen (DiFabio 2005). ${ }^{3}$ Dazu soll auch hier beigetragen werden.

\subsection{Kernproblem}

Besonders aktuell ist die Nobelpreisvergabe 2017. Sie ist ein erklärter Protest gegen den homo oeconomicus, obgleich der Preis ausgerechnet an einen Forscher aus Chicago gegangen ist, wo die Übertreibungen dieser Lehre beheimatet waren (Joffe 2017; Thaler und Sunstein 2008). Hinzu kommen zahlreichen gesetzgeberischen Initiativen auf Gebieten des Bank-, Kapitalmarkt- und Insolvenzrechts ${ }^{4}$ und sogar auch einige Ansätze der Rechtsprechung zur Sittenwidrigkeit in bestimmten Fallgruppen rücksichtloser Risikotransfers (BGHZ 173, 246 - Trihotel) und zu eigenkapitalersetzenden Gesellschafterdarlehen (BMF 2010). Doch fehlt es - sieht man einmal von einigen älteren wirtschafts- (Schumpeter 1954; Müller-Armack 1970, S. 19 ff. $)^{5}$ und sozialwissenschaftlichen (Weber 1980, S. 329; ders. 1998, Band 1 S. 94 f.) Versuchen ab - an der genaueren Erforschung des Wechsels von Menschenbildern und anthropologischen Paradigmen, die in der Vorbereitung marktwirtschaftlichen Denkens eine tragende Rolle gespielt haben und deshalb für ethische Prinzipien der Humanität heranzuziehen sind ${ }^{6}$

Zwar haben sich neuerdings humanitätsbetonte Entscheidungslehren und pragmatische Theorien des Individu-

2 Allgemein ökonomisch vgl. Sen (2011); Shiller (2012); wirtschaftsethisch Koslowski (1982); Homann und Suchanek (2005); zur Verantwortungsethik vgl. Suchanek und Lin-Hi (2006); Homann und Gruber (2014); Enste (2015) mit weiteren Nachweisen; Worsdörfer und Dethlefs (2010), S.75 ff.; z.T. kritisch Meyer (2014), S. 387, 407 ff. (zur z.T. nicht weit entfernten Alternative „moderner Sozialpsychologie“ ebd., S. 408 f.); vgl. auch Frey (1997).

3 Jüngst als politisches Konzept des französischen Präsidenten Macron: gesamteuropäische Kultur als „soziales Bindemittel“ zur Abwehr populistischer Ausländerfeindlichkeit und Gewaltbereitschaft.

4 Zum Finanzmarktstabilisierungsgesetz (FMStG) vgl. nur Seibert (2010), S. 2525 ff.; zum MoMiG vom 23.10.2008, BGBI. I, 2026; vgl. Herrmann und Roth (2008), Band 2, IV.3d.

5 Auch Röpke (1979); Schmitz (Hrsg., 1982); Lachmann (1989); zu Inkonsequenzen der in Salamanca begründeten Theorie des freien Marktes mit Preisbildung durch Angebot und Nachfrage vgl. von Hayek, zitiert nach de Roover (1955), S. 161 ff. und S. 170; neuere Versuche aber bei Sedlacek (2013); Meyer (2014), S. 387 ff.; ders. (2002); Frey (1997).

6 Wertvolle Ansätze bei Homann (1999), S. 335; Homann und Suchanek (2000); zur theologischen und kulturwissenschaftlichen Ethik vgl. Huber (2012), S. 17 und 112, passim; DiFabio (2005), S. 13 und 26 f.; Horn (2007), S. 52. alismus $^{7}$ sowie Ethikkonzepte der corporate governance ${ }^{8}$ als recht erfolgreich erwiesen (Wieland 2007, S. 71, passim; Beiträge in Hopt und Teubner 1985) und z.T. sogar ins geltende Recht Eingang gefunden. ${ }^{9}$ Doch ist zum einen das pragmatistische Anliegen schon wegen des Scheiterns älterer Vorläufer ${ }^{10}$ reichlich angreifbar; und zum anderen scheint die Forschung zur GovernanceEthik immer noch zu stark auf die Mathematisierung der Steuerungsanreize und die Binnenstrukturen der Aktiengesellschaft und der Unternehmenskultur (corporate governance) ausgerichtet. ${ }^{11}$ Zudem leiden die genannten Forschungsrichtungen noch an Defiziten zur Historie individualistischer Menschenbilder und der dazu gehörigen ethischen Publizitätsstrukturen.

Im Folgenden soll dazu v.a. dadurch beigetragen werden, dass nach den Grundvoraussetzungen wettbewerblichen Wirtschaftens im individualistischen Menschenbild und dessen Paradigmenwechsel in der Reformationszeit geforscht wird. ${ }^{12}$ Fragt man, weshalb ausgerechnet theologische Geschichtsforschung bemüht werden muss, um zur modernen Wettbewerbsethik beizutragen, so ist es wohl nicht ganz zureichend, auf die Wertefundierung der Anreizökonomik abzustellen, wie sie neuerlich v.a. von wirtschaftspsychologischer Seite betont wird (Thaler und Sunstein 2008) und in der Preistheorie und rechtstheoretischen Entscheidungslehre schon seit Jahrzehnten gefordert worden ist (Diller 2007, S.164 ff. zu 4.7.4.2; Herrmann 1977). Denn letztlich muss man sich vor Augen halten, dass es nun einmal Theologien der Glaubensrechtfertigung waren, die zur Entstehung des individualistischen Menschenbildes und des damit verbundenen Wettbewerbsdenkens geführt haben. Das ist, was Calvin angeht, schon seit Max Webers (1985) Kapitalismusthese herrschende Meinung, gilt aber natürlich auch für die Gegenansicht, nach der nicht der Protestantismus, sondern

7 Vgl. von der Pfordten (2011), S. 33 ff.; ähnlich Nida-Rümelin (2011), S. 109 ff.; ders. (1997), S. $175 \mathrm{ff}$.

8 Vgl. nur Wieland (2007), S. 71, passim; ders. (2001), S. 8 und 29; für mehr geistesgeschichtliche Akzente vgl. Homann (1999), S. 335; Homann und Suchanek (2000); Beiträge in Hopt und Teubner (Hrsg., 1985) mit weiteren Nachweisen.

9 Vgl. § 161 AktG i.V. mit dem Corporate Governance-Kodex (laufende Updates unter www.dcgk.de)

10 Zum älteren Pragmatismus relativistischer Nützlichkeitsphilosophie vgl. James (1994); zum Neopragmatismus vgl. Schubert et al. (2010); z.T. kritisch Montenbruck (2013); gegen die Instrumentalisierung in der Wettbewerbstheorie vgl. Immenga (1976); neuerlich Wieland (2001) mit Bezug auf die Governance-Ethik.

11 Vgl. nur Wieland (2007); ders. (2001); zum Kartellrecht vgl. Hopt (2012), S. 290 ff.; zu Teilaspekten vgl. Herrmann (2009), S. 815 ff.; ders. (2006), S. $101 \mathrm{ff}$.

12 Für eine ausführlichere Untersuchung zum Thema sei auf die Monographie des Verfassers (2018) verwiesen. 
die Spätscholastik und insbesondere die berühmte Schule von Salamanca als geistige Grundlage für die Wettbewerbslehre des Adam Smith und der auf inm aufbauenden Fortentwicklung des Ordo-Liberalismus anzusehen sein soll (Höffner 2014; Franco 2016). Will man die weitere Entwicklung bis heute verstehen, so muss man sich auf die vernunftethische Säkularisierung der Glaubensrechtfertigung einlassen, die nicht nur bei Thomasius, sondern auch bei Adam Smith und Immanuel Kant klassisch formuliert worden sind. Dass der Gegensatz zu Verdienstgerechtigkeit bis heute nicht verloren gegangen ist, beweisen nicht nur die nicht enden wollenden Streitfragen zu Vorstandsboni, Turnierprämien von Profisportlern und dergleichen, sondern es geht auch um die Formel: Leistung muss sich wieder lohnen! Deren Berechtigung ist durchaus auch unter protestantischen Aspekten prominent, wenngleich auch nicht unangreifbar (Huber 2012, S. 28), anerkannt. Auch die übertriebenen Risikosteigerungen und Gewinnerwartungen von Banken und Versicherungen in den Finanzkrisen werden in diesen Sinnzusammenhang eingeordnet (Huber 2012, S. 112). Ja, sogar das ansonsten hochmoderne MoMiG $^{13}$ ist zum Eigenkapitalersatz von Gesellschafterdarlehen nicht frei von rechtsethischen Widersprüchlichkeiten, die zwar keinen Eingang in die Gesetzbegründung gefunden haben, wohl aber rechtsethischer Hervorhebung und Kritik bedürfen.

\subsection{Methodisches und Aufbau der Untersuchung}

Zur Maßgeblichkeit ethischer Orientierungen wird an das wissenschaftliche Konzept von Thomas Kuhn angeknüpft, das - nicht erstmals, aber wissenschaftstheoretisch besonders fundiert - auf paradigmatischen Wechsel von Welt- und Menschenbildern abstellt (Küng 1973). Mit Küng u.a. wird das Kuhnsche Modell kopernikanischer Revolution der Astronomie auf den anthropologischen Wechsel vom mittelalterlichen Menschenbild des Kirchenchristen zum neuzeitlichen Individualismus übertragen (Küng 2006, S. 91 ff.). Ansätze dazu gibt es übrigens schon bei Kant, der seine Erkenntniskritik im Zusammenwirken mit der des Descartes als philosophisch-kopernikanische Revolution bezeichnet hat (zitiert nach Horn 2016: „kopernikanische Tat“).

Beinahe unbemerkt für die rechtsethische Forschung, aber längst vielfach von theologischer Seite anerkannt (Herms 2001; Stock 2011, S. 119) ist eine grundlegend neue Sichtweise der lutherischen Rechtfer-

$13 \S \S 19$ Abs. 2, 135 InsO v. 18.10.2008 i.d.F. des MoMiG, BGBI. I, S. 2026; Neufassung aber v. 1.1.2014, BGBI. I, S. 1982. tigungslehre, die nicht nur die Kritik am metaphysischen Philosophiekonzept der Scholastik betont, sondern deren Kern in der sogenannten Kontingenzlehre sieht. Damit ist der eigentliche Grund für den Wandel zur individualistischen Anthropologie entdeckt, wenngleich die nötigen Forderungen für die säkulare Moralphilosophie und zu deren Auswirkungen auf die Wettbewerbsethik bisher, soweit ersichtlich, noch von Niemandem gezogen worden sind. Die Kontingenzphilosophie betrifft aber eine tiefgreifend erkenntniskritische Einschätzung, die eine Art Risikolehre menschlichen Lebenssinn eröffnet. Aus der kantischen Vernunftethik ist schon länger bekannt, wie schwerwiegend kontingente Bedingtheit menschlicher Erkenntnis zum Menschenbild des Individualismus beiträgt. ${ }^{14}$ Aber erst neuere Forschungen haben gezeigt, dass hier entscheidende Weichen schon in Luthers Rechtfertigungslehre gestellt wurden (nochmals Herms 2001; Stock 2011, S. 119; Groß et al. 2011). Ist dies aber so, so sind die Auswirkungen auf das individualistische Menschenbild der Auflärung bis hin zu Kant vor allem unter Aspekten der Kontingenzlehre interessant, ja man wird zu fragen haben, ob auch moderne Vernunftkritik, wie die der schon erwähnten Demokratietheorie zum Politikversagen (Jann und Wegrich 2004, S. 198; Sturm 2009, S. 18 f.) und die wettbewerbliche Kritik von Wissensanmaßung über Marktergebnisprognosen (v. Hayek 1967) auf eben dieser erkenntnisphilosophischen Kontingenzannahme beruhen. Was daraus zudem für die Ethik der Risikotheologie und Risikophilosophie folgt, ist noch sehr weitgehend unerforscht (vgl. Schilling 2017, S. 402 f., 643 f.; Linke 2005, S. 17). Es gibt lediglich erste Hinweise auf Trends der amerikanischen Verantwortungsethik (Rawls 1971, S. 60 f.; Mounk 2017, passim) und corporate governance (Wieland 2007), denen im Folgenden nachzugehen sein wird.

Andererseits gibt es zahlreiche Ansätze einer Rechtsethik, die nicht in die Tradition der GovernanceEthik amerikanischer Herkunft gehört, sondern viel direkter mit der europäischen Entwicklung wettbewerblicher Steuerung verbunden ist (Reuter 1973; Horn 1979). Man geht deshalb wohl nicht allzu viel wissenschaftliches Risiko ein, wenn man Luthers Theologie - soweit ersichtlich, erstmals - auf paradigmatische Wurzeln der Verantwortungsethik wettbewerblichen Risikomanagements hin untersucht. Das soll hier geschehen.

Dabei genügt es eigentlich nicht, mit der Reformationszeit zu beginnen. Schon Adam Smith hat als Begründer des Wettbewerbsdenkens nicht nur die calvinistische und lutherische Rechtfertigungstheologie im

14 Zur Selbsteinschätzung Kants als paradigmenhistorischer Revolutionär s.o. 
Auge gehabt, sondern auch auf die berühmte invisible hand als Formal alttestamentlicher Theologie abgestellt (Smith 1994). Deshalb beginnt der vorliegende Beitrag mit einerallerdings nur stichwortartigen Zusammenfassung - der Handtheologie des Alten Testaments (zu II) und schreitet fort über die Scholastik bis zu Luther (III.), Thomasius und Kant (IV.), die beide zu den protestantisch motivierten Starphilosophen der Aufklärung gehören. Doch damit kann die Abfolge großer Denker der Traditionsgeschichte dann auch beendet werden, da die Untersuchung auf die highlights von paradigmatischer Bedeutung begrenzt bleiben soll. Nur wenige Schlussbemerkungen sind zum amerikanischen Kantianismus bei Rawls und dessen Auswirkungen auf die aktuelle Verantwortungsethik des US-Wirtschaftsrechts angebracht (V.1). Abschließend werden einige Fallgruppen zum aktuellen Recht sittenwidriger Rechtsgeschäfte und Schädigungen angesprochen, in denen sich der hier erarbeitete Ansatz ethischer Verantwortungsbereitschaft zu bewehren haben wird (V.2).

\section{Wettbewerbliche Menschenbilder im Altertum und Mittelalter}

Die wohl bekannteste Formulierung des Begründers ökonomisch-wettbewerbswissenschaftlichen Denkens, Adam Smith, geht dahin, dass es die invisible hand sei, die den Funktionszusammenhang von individueller Vorteilssuche und gesamtwirtschaftlicher Wohlfahrt herstelle. Bedenkt man, dass Adam Smith im Rahmen seiner Lehrtätigkeit auch Vorlesungen zur Moralphilosophie zu halten hatte, und dass er keineswegs, wie früher zum Teil angenommen (Marx 1968, S. 645 f.), ein überzeugter Atheist war, so liegt es nahe anzunehmen, dass inm der alttestamentliche Bezug auf Geschichten der Hand Gottes (jad Jahwe) geläufig war und dass er hierauf auch bewusst angespielt hat. Zwar ist schon die Geschichte vom Sündenfall des 1. Buches Mose, Kapitel 3, äußerst lehrreich für Fragen der wettbewerblichen Rivalität und freien Persönlichkeitsentfaltung (Herrmann 2018). Doch wird hierauf bei Smith nicht ausdrücklich Bezug genommen, so dass sich das Folgende auf die Vorstellung von der unsichtbaren Hand Gottes in den Davidserzählungen konzentriert.

Nach der Richterzeit war es für den Glauben im Alten Testament ein Problem, neben der Allmacht Gottes einen absoluten Herrscher über das Volk Israel legitimiert zu sehen. Das Handauflegen oder die Hand von jemandem wieder wegnehmen war deshalb als Legitimation bzw. Entmachtung von oberster Stelle gedacht und insofern eine der Stellvertreterstellung beim Opferritus verwandte Erzähltradition. ${ }^{15}$ Denn auch beim Handauflegen und dem Entziehen der Hand Jahwes von Saul, David und Salomon spielt die Verzeihlichkeit bzw. Unverzeihlichkeit von Sünden eine tragende Rolle. ${ }^{16}$

So sind Sauls und vor allem Davids schreckliche Missetaten bis hin zu Mord und Vergewaltigung häufig gar kein Grund zur Aufkündigung des Segen Gottes, den er einmal durch das "Fallen in die Hand des Herrn" (2. Sam 24, 14) erteilt hat, bei dessen Entzug aber die „Hand des Herrn wider euch und euren König sein“ kann (1. Sam. 12, 15). Dem König David wird sogar gesagt: „... Von Frevlern mag Frevel ausgehen, aber meine Hand soll nicht wider dich sein." Die neuere David-Exegese geht sogar dahin, dass das Alte Testament sich vor allem deshalb so ausführlich mit den Todsünden Davids befasst, weil gezeigt werden soll, wie weit die Gnade des Herrn auch bei Berichten rivalisierender Machtgruppen (Würthwein 1947; Nitsche 2002, S. 218 ff. mit weiteren Nachweisen) von schlimmsten Missetaten reichen kann.

Im Mittelalter war für das Menschenbild im Christentum und die Stellung des Menschen in der Kirche lange Zeit die Schrift des Augustinus (2012) vom Gottesstaat maßgebend, die einen wichtigen Grundpfeiler zur Trennung von Staat und Kirche und damit zur Begründung päpstlicher Macht errichten sollte. ${ }^{17}$ Doch war es erst im 13. Jahrhundert Thomas von Aquin vorbehalten, die Glaubenslehre in Anknüpfung an die lange verschollenen aristotelischen Quellen maßgebend in einem kirchenchristlichen Sinn zu verändern. Auch Thomas ging es wie Aristoteles - um die Verwirklichung wahren Menschseins, die aber - anders als in platonischer Tradition - nicht außerhalb der Erscheinungswelt in den Dingen an sich erkannt werde, sondern im Menschen selbst vorhanden sei. ${ }^{18}$ Der Kausalität von natürlichen Geschehensabläufen wurde die Finalität der Beziehung aller Dinge auf Gott gegenübergestellt. Die aristotelische Epagogik wurde deshalb als Verinnerlichung im Sinne einer Art Substanzmetaphysik verstanden. ${ }^{19}$ In der mittelalterlichen Weiterentwicklung fasste man die Gottesvorstellung als oberstes Sein in einer naturhaften Pyramide von Seinsstufen und definierte Gott als summum bonum im Sinne einer höchsten Zweck- und

15 Vgl. nur 1. Sam. 12, 15 „jad Jahwe“ (Hand des Herrn); 2. Sam. 24, 14, 16 f.: Hand des Herrn (jad adonai)

$16 \mathrm{Zu}$ den Gegensätzen des Deismus und der prästabilisierten Harmonie eine Leibnitz u.a. siehe unten (zu V. 4a).

17 Dazu und zu Folgenden vgl. Küng (2006), S. 343 ff.; stark vereinfachte Darstellung von Precht (2013), S. $391 \mathrm{ff}$.

18 Vgl. Thomas (1977), zu 1, 1a.1; 1-2 a.2; vgl. Schwöbel (2000), Sp. 1116.

19 Dazu und zum Folgenden siehe Enskat (2002), Sp. $1171 \mathrm{ff}$. 
Zielstrebigkeit der Welt. ${ }^{20}$ Ersichtlich handelt es sich auch bei dem Menschsein in dieser Seinsstufung nicht um das konkrete Individuum, sondern um eine übergeordnete Wesensvorstellung. Der Mensch in dieser Sicht war auch als gläubiger Christ niedrig.

Auch zur Zeit Luthers blieb es in der altrömischen Theologie beim anthropologischen Paradigma des Spätmittelalters, wie sich etwa an der Ansicht des Widersachers Luthers, Johannes Eck, zeigt. Bei Eck geht es im Wesentlichen um dessen im Jahr 1519 erschienen Traktat über die Werkgerechtigkeit und um seine Leipziger Disputation mit Luther im selben Jahr. Sowohl im Traktat als auch in der Disputation bezog Eck sich auf die aristotelische Lehre vom freien Willens des Menschen, wie sie in der Rezeption des Thomas von Aquin als herrschende Meinung der Spätscholastik bekannt war. Eck greift auf die scholastischen Grundlehren zurück und hebt dabei den freien Willen jedes Menschen hervor, um den Gegensatz zu Luther herzustellen. ${ }^{21}$ Implizit wird dadurch allerdings eine stärker auf das Individuum bezogene Anthropologie übernommen. Doch wird sowohl die Lehre priesterlicher Stellvertretung nicht in Frage gestellt als auch eine enge Verbindung zur Kurie gehalten (Schilling, a.a.O, Fn. 21; und zudem wird hoch umstritten verhandelt, wie Luther über das Priestertum aller Gläubigen dachte. ${ }^{22}$ Dazu sogleich mehr.

\section{Umbruch des Menschenbildes bei Martin Luther}

Soviel zum alten Paradigma des Spätmittelalters und dessen Vorläufern. Wie kam es zu Beginn des 16. Jahrhunderts zur entscheidenden Durchsetzung des Paradigmenwechsels? Und welche Unterschiede lassen sich feststellen, die - anders als bei den schon aus früherer Zeit bekannten spätscholastischen Kritikern des Thomas - eine allgemeinere Etablierung der neuen Lehren ermöglichten?

\subsection{Zur Spätscholastik und zu Schule von Salamanka}

Für die revolutorische Struktur ist besonders beachtlich, dass vor dem entscheidenden Umbruch zur Neuzeit

20 Vgl. nur den 5. Gottesbeweis des Hauptwerkes der Summa Theologiae (1276-1273), deutsch-lateinische Ausgabe 1977, vgl. Horn (2016), Rdn. 290.

21 Vgl. schon Brecht (1981), Bd. 1, S. 205; Schilling (2012), S. 189 f., 196 mit weiteren Nachweisen.

22 Näher und vor allem in aller Öffentlichkeit, allerdings erst in der Schrift Luthers (1520), An den christlichen Adel deutscher Nation. anderwärts zahlreiche Streitigkeiten zum Ausbruch kamen, die das paradigmatisch Neue vorbereiten. (Kuhn 1996, S. 210 ff.). Das gilt schon für den ältesten Kritiker des Thomas aus Schottland, Duns Scotus, der vor allem das metaphysische Denken angriff und sich für bloßes Erfahrungswissen stark machte. ${ }^{23}$ Aber es fehlt an generellem Kontingenzdenken. Wissen aus Erfahrung ist nach Duns nicht nur Messen und Wiegen, sondern auch die Universität des Seins, d.h. Gott ist zwar nicht im Wege der analogia entis des Thomas erkennbar, aber er hat mit dem Menschen das Sein gemeinsam, so dass seine Existenz nicht nur möglich, sondern erfahrbar ist (Rieger 2004). Damit verbunden ist auch in gewissem Umfang ein Sinnerleben von Welt.

Stärker Thomas-harmonisierend waren indessen die Ansichten der Schule von Salamanka, die dennoch ebenfalls ein deutliches Frühlingserwachen des Spätmittelalters bedeuten. Einer der führenden Theologen und Dominikaner, Francisco de Vittoria (1492-1546), gilt wegen seiner Schriften als „Vater des Völkerrechts" und wurde schon von Hugo Grotius zur Begründung seines ebenfalls grundlegenden Völker-Naturrechts wiederholt als anerkannte Autorität herangezogen. ${ }^{24}$ Dreh- und Angelpunkt des jus gentium von Vitoria war das eigentlich ganz unaristotelische und jedenfalls völlig unthomische Menschenbild, wonach die Eingeborenen Amerikas nicht wegen kindlicher Intelligenz besonderer Führung durch die Europäer bedürften, sondern Anteil an der gleichen menschlichen Natur haben. Dennoch hat schon Francisco Suárez die Meinung vertreten, dass politische Macht nicht dem einzelnen Individuum zukomme, sondern dem Volk als Ganzem gebühre. Schon deshalb, aber auch wegen der laufenden Harmonisierung mit thomistischer Lehre kann man den Anhängern der Schule von Salamanca kaum den Durchbruch zum Individualismus im Sinne eines Paradigmenwechsels zurechnen.

\subsection{Erasmus als Mit-Reformator und Medienstar}

Zunächst ist der akademisch führende Kopf der Zeit zu erwähnen, der Humanist und der erste Medienstar aus Rotterdam, Erasmus, dessen Lehren geraume Zeit vor dem Bekanntwerden Luthers enorme Verbreitung fanden, aber wegen weitgehend fehlender Kritik am

23 Dazu und zu Folgenden vgl. nur Duns Scotus, Ordinatio, a.A., zitiert nach Rieger (2004) zu Entsprechungen bei Ockham und dessen Exkommunikation (1328) vgl. Herrmann (2017).

24 Vgl. schon von Stechau (1848), Die Vorläufer des Hugo Grotius, zitiert nach Wieacker (1967), S. 284 Fn. 14 mit weitern Nachweisen; gegen Überschätzung dieses Einflusses allerdings Welzel (1962), S. 123 f. 
Klerus und am Papst weit weniger Anstoß erregten. Er gilt als „geistiger Ziehvater“ Luthers. ${ }^{25}$ Melanchthon nennt als jüngerer Zeitgenosse seine Lehrer Reuchlin, Erasmus und Luther zwar als gleich bedeutende Theologen der Erneuerungsbewegung, räumt aber Erasmus einen gewissen Vorrang ein. Dieser habe als erster Theologe die typisch humanistische Forderung begründet, dass man zu den Quellen der Bibelüberlieferung vorzustoßen habe. ${ }^{26}$ Daraus folgte natürlich, dass der Kirche und den päpstlichen Beschlüssen nicht länger Vorrang vor der Schrift einzuräumen und immer der Schriftauslegung der Vorzug gebühre. Doch zog diese Folgerung eben erst Luther und im Lager der Humanisten nicht Erasmus, sondern erst sein Schüler und Luther-Freund Melanchthon in seinen (weitgehend selbst aufgestellten) Disputationsthesen zum Baccalaureus biblicus im Jahr $1519 .{ }^{27}$

Nicht minderbedeutend war die Ansicht des Erasmus, der Mensch habe einen freien Willen. Dazu verdient jedoch die Schrift über das "Lob der Torheit“ (Erasmus von Rotterdam 1975, S. 1-211) Hervorhebung, weil hier unmittelbar an die oben erwähnten Vorbereitungslehren von Duns Scotus bis Ockham angeschlossen und daraus hergeleitet wurde, dass der Mensch nicht von sich aus erkennen könne, was Gott wohlgefällig sei. Jedoch zog Erasmus daraus, wie auch schon die erkenntniskritischen Theologen vor inm, nicht die radikale Folgerung, der Mensch werde wegen ebendieser begrenzten Vernunftmöglichkeiten nicht wegen seiner Werke gerechtfertigt. Vielmehr komme der Mensch zur Vervollkommnung seiner Freiheit, indem er zu „wahrer Freiheit“ hindurch finde, auch wenn diese letztlich von Christus gebracht worden sei. ${ }^{28}$ Hierin und in der dadurch ermöglichten Eigenverantwortlichkeit des Menschen liege der Sinn menschlichen Lebens; denn, wie Schilling sagt, „ohne Willens- und Entscheidungsfreiheit konnte er [Erasmus] menschliche Verantwortung weder philosophisch noch theologisch denken" (Schilling 2012, S. 400). Mit dieser Ansicht, die sich in Ansätzen schon als individualistische Verantwortungslehre der Neuzeit bezeichnen lässt, sah sich der Humanist imstande, für die Erneuerung von Kirche, Theologie und Volksfrömmigkeit einzutreten, wohl gemerkt eine Erneuerung der Kirche von innen heraus, und nicht gegen die

25 So etwa Küng (2006), S. 635

26 Melanchthon, MBW 40.4 = MBW T 1, 101.44-47; dazu Scheible (2010), S. 10; vgl. Schilling (2010), S. 396: „führender Kopf der Humanisten".

27 Melanchthon (1977), Thesen 16-18.

28 Erasmus, zitiert nach Schilling (2012), S. 399; zum Gegensatz der lutherischen Soteriologie extra nos und der damit zusammenhängenden Kontingenzlehre s.u. zu 3.
Kurie, sondern in laufend wiederkehrendem Einvernehmen mit dieser. ${ }^{29}$

\subsection{Luthers individualistisches Paradigma und reformatorische Ethik der Verantwortlichkeit}

Das Wirken im öffentlichen Meinungskampf mit der Kurie und im Wissen um die alleinige Glaubensrechtfertigung blieb Martin Luther als dem führenden Kopf der Reformationsbewegung überlassen. Zunächst zu den bekannten Grundlehren.

a. Rechtfertigung allein aus Glauben und Kontingenzansatz. Luther führte der Umgang mit den biblischen Quellen zu der Erkenntnis, dass eine über die kanonischen Texte hinausgehende Offenbarung dem Menschen verschlossen sei. Man könne sich lediglich auf biblische Lehren vom „verborgenen Gott“ zurückziehen, wie sie etwa im Buche Hiob zum Ausdruck gelangten. Der Mensch stehe, wie seinerzeit Hiob, bereits auf Erden vor Gott „, in sua absolute maiestate“, doch sei es inm auch im Anschluss an Ockhams mit der Bibel übereinstimmende Lehre vom verborgenen Gott ${ }^{30}$ vollkommen unmöglich, Gottes Gerechtigkeit nach irdischen Maßstäben von Gut und Böse einzuschätzen. Folglich komme nur die Rechtfertigung „allein aus Glauben" (sola fide) in Betracht (Luther 2000-2007, WA 40 II; 328, S. 1 f.). Die Glaubensgerechtigkeit wird dabei nicht als physiologisch gedachter Rechtfertigungs- und Humanisierungsprozess übernatürlichen Ursprungs verstanden, der innerhalb des Menschen stattfinde, wie es noch Thomas gelehrt hatte. Zwar handele es sich um eine der Seele eingegossene Gnade (gratia infusa), die aber allein in Jesus Christus sozusagen von außen her (extra nos) offenbar geworden sei. Mit dem so gefassten christologischen Aspekt wird die aristotelischthomistische Philosophie der wahren Menschwerdung von innen her grundlegend verlassen, auch wenn die gratia infusa zugleich eine Kraft ist, die nicht allein von außen her infundiert ist, sondern auch vom Inneren des Menschen her wirkt. Die Loslösung vom aristotelischen Denken ist in dieser Hinsicht zwar nicht ganz vollständig; ${ }^{31}$

29 Vgl. McConica (1991), zitiert nach Winkler (1999), Sp. 1380 ff. unter Hinweis auf die spanische Erasmus-Rezeption in der Schule von Salamanca und bei den spanischen Schwärmern.

30 Dazu vgl. näher Küng (2006), S. 610; Schwöbel (2000), Sp. 1117

31 Zustimmung Luthers zu zahlreichen Aristotelismen bei Melanchthon vgl. Scheible (2010), S.20 f. Auch im Abendmahlsstreit nahm Luther eine Zwischenstellung ein, die zwar auf scharfer Ablehnung der thomistischen Transsubstantiationslehre beruhte, aber mit der Vorstellung einer Consubstantiation doch dem aristotelisch-thomistischen Substanzdenken verhaftet blieb; vgl. Schilling (2017), S. 247 ff.; zur Vermittlung durch Melanchthon vgl. nochmals Scheible (2010). S. $23 \mathrm{ff}$. 
und doch ist der Umbruch zur Rechtfertigung im Sinne bloßer Glaubensgewissheit das entscheidend Neue.

Hieran knüpft die bereits eingangs angesprochene Überlegung an, Luther sei aus der Kontingenzlehre heraus zu verstehen, die später Kant als transzendentalphilosophischen Kern seines sog. Regelskeptizismus im Sinne einer systematischen Fortentwicklung des cartesischen Subjektivismus und Individualismus herausgestellt hat. Dazu an dieser Stelle nur der folgende Denkansatz: Kant sah jede Sinnvorstellung von Welt und Menschenleben als kontingent an, d.h. als bloß unerweisliche Möglichkeit erfahrungsüberschreitender Rationalität (Transzendenz). Inhaltliche Aussagen dazu lassen sich nur mit Bezug auf andere Elemente transzendentaler Sinnsuche des Menschen machen, wobei ebendieses Bestreben des Menschen nach Sinnerklärung im Leben unausweichlich mit dem Menschsein verbunden sei. Das geht natürlich weit über die theologische Gedankenwelt Luthers hinaus, hat aber mit dieser gemeinsam, dass auch das Denken des Menschen, der Welt müsse irgendeine Gerechtigkeit innewohnen und eine Rechtfertigungslehre zu entnehmen sein, nichts Anderes ist als ein solcher transzendentalphilosophischer Bezugspunkt. Man denkt Gott und ein jüngstes Gericht, um damit Lebenssinnhaftigkeit zu begründen

Luther bezog sich für diese Annahme auf die biblischen Erzählungen vom Sühnetod Christi am Kreuz und sah darin eine Wortoffenbarung, die den Anforderungen des humanistischen Geschichtsbild recht gut entsprach. Mehr Philosophisches war dazu aus Luthers Sicht eigentlich nicht nötig, da mit der These der ausschließlichen Autorität der Schriftoffenbarung die gesamt Metaphysik des Thomas von Aquin falsifiziert erschien. Nur relativ untergeordneten Rang nahm deshalb bei Luther die Lehre vom verborgenen Gott ein (deus absconditus), die vor inm schon Andere, wie gezeigt, zur Thomas-Kritik verwendet hatten. Aber selbstverständlich war dem Reformator dieser theologische Ursprung kontingenztheoretischer Lehren und individual-anthropologischen Denkens sehr gut bekannt. Nur blieb vorerst noch lange die Einsicht verdeckt, dass Kontingenz nicht nur im Glauben gilt, sondern auch die Affektenlehre des Vernunftrechts bestimmt und transzendentalphilosophisch grundlegend ist. Auf Beides ist im Folgenden v.a. zu Thomasius und Adam Smith sowie zu Kant ausführlich zurückkommen. Und doch ist der kontingenztheoretische Ansatz Luthers bereits Grund genug, die Neuerungen als so grundlegend anzusehen, dass man darin den Paradigmenwechsel zur Neuzeit erkennen kann. ${ }^{32}$

Zur Ethik folgt daraus, wie sich eigentlich von selbst versteht, dass der Christ nicht fröhlich darauf lossündigen kann, sondern dass er im Glauben lebt, d.h. - modern gesprochen - verantwortungsbereit ist, auch wenn er keine guten Werke vor Gott vollbringen kann. Dafür ist natürlich in erster Linie auf Luthers scharfe Predigten gegen die Gräuel der Ritter- und Bauernaufstände zu verweisen, die während seines Zwangsaufenthaltes auf der Wartburg losbrachen (Schilling 2012, S. 247 ff.). Auf die damit zusammenhängenden Lehren zum verantwortungsbereiten Leben im Glauben wird im übernächsten Abschnitt gesondert eingegangen. Doch zunächst zur Frage der freien Willensbildung, die für das wettbewerbsethische Problem der Handlungsfreiheit auf Märkten mit Preis- und Qualitätswettbewerb von erstrangiger Bedeutung ist.

b. Zum Streit Erasmus-Luther über die Willensfreiheit und zur Bedeutung des Humanismus. Wie schon erwähnt, hat Erasmus die Willensfreiheit vertreten, dies aber im Gegensatz zu Eck, Tetzel u.a. nicht mit einer Kollektivlehre zur Legitimation der Werkgerechtigkeit, klerikaler Machterhaltung und thomistischer Metaphysik verbunden, sondern auf seine individualistische Verantwortungslehre gestützt und darin ohne grundlegenden Konflikt mit den Anhängern Roms eine „humane Perfektionierungsmöglichkeit“ entdeckt (Schilling 2012, S. 401). Luther fand daran nach anfänglichem Zögern nichts Richtiges und verfasste seine Gegenschrift „De servo arbitrio“ (Vom verknechteten/unfreien Willen), die im Jahr 1525 erschien. Es sei eine viel zu optimistische Illusion, eine eingeborene Vernunft mit der Fähigkeit zum Guten anzunehmen und von dieser eine selbstständige Erlangung des himmlischen Heils zu erwarten. Der kontingenztheologische Bezug zur Versöhnung Gottes am Kreuz Jesu Christi werde grundlegend verkannt. Stattdessen wird eine wenngleich für das weltliche Naturrecht nur mit Einschränkungen erhebliche (Scheible 2010, S. 298 f.) Anthropologie unfreien Willens, wie folgt, begründet:

Luther teilt die zu Erasmus erläuterte Morallehre erneuerter Herzensfrömmigkeit, die wir dort als Lehre von der Eigenverantwortlichkeit bezeichnet haben. Sie wird in dogmatischer Anbindung an die Lehre von Gesetz und Evangelium behandelt. Gesetzlichkeit menschlichen Handelns wird nicht zur Voraussetzung des Evangeliums der Erlösung, wie in der Gegenansicht Ecks, sondern sie sei der evangelischen Botschaft lediglich gegenüberzustellen. Wo dies allerdings als Befreiung von der Höllenangst aufgenommen wurde

32 Vgl. Küng 2006, S. 602 ff.; dazu schon oben zu II.1. 
und sogar zum Teil als Berechtigung zu Mord und Todschlag durch Ritter und Bauern gedeutet worden ist, hat Luther dem heftig widersprochen. Der moderne, schon eingangs erwähnte Streit um die Rechtfertigung zu billigen Bedingungen findet in dieser Gegenüberstellung seine Lösung: ${ }^{33}$ Angstbefreiung ja, aber keine Gnade ohne Glauben und gläubige Befolgung der zehn Gebote. Keine Vergesetzlichung, wie Jesus sie gegen die Pharisäer des Judentums seiner Zeit bekämpft hat, sondern Herzensfrömmigkeit in Gläubigkeit und in darin geeinter Gemeinschaft aller Gläubigen, die vor Gott vollkommen gleich seien.

Besonders pointiert stellt Luther die Freiheit „Von“ etwas derjenigen „für“ etwas gegenüber. Freiheit „von“ führt dazu, dass der Christ „niemandem untertan“ ist, die Freiheit „für" besagt geradezu das Gegenteil, wie in dem berühmten Zitat: „Ein Christenmensch ist ein freier Herr über alle Dinge und niemandem untertan [...] und ein dienstbarer Knecht aller Dinge und jedermann untertan“ (Luther 2007, S. 47). Der langjährige EKDRatsvorsitzende Huber hat in dieser Gegenüberstellung ein lutherisches Freiheitspostulat gesehen, das bis heute als „verantwortete Freiheit" von hervorragender Aktualität sei (Huber 2012, S. 16 ff., 32 ff.). Der Christ ist nach Huber bis in die heutige Zeit nicht nur unfrei und deshalb erlösungsbedürftig in evangelischer Botschaft und ohne Voraussetzungen von Gesetzestreue, sondern er wird befreit zu gutem Handeln im Glaube und in Verantwortlichkeit. Das gilt natürlich nur in Bezug auf christliche Ethik, zeigt aber auch sehr deutlich, inwieweit Scheinwidersprüche der lutherischen Lehre von der Willens(un)freiheit wenigstens teilweise auflösbar sind. Sie ist im kontingenten Zusammenhang mit der biblischen Botschaft vom Kreuzestod zu sehen. Erst spätere Verallgemeinerungen vernunftkritischen Denkens werden erweisen, wie wegwesend die lutherische Rechtfertigungslehre für die Entwicklung des modernen Naturrechts bis hin zur Wettbewerbstheorie eines Adam Smith und bis heute gewesen ist (Schilling 2017, S. 403).

Man kann in dieser revolutionär-individualistischen Dimension Luther'scher Theologie geradezu den wichtigsten Grund dafür sehen, dass sich seiner Lehre allen voran die wohlhabenden Kaufleute und Patrizier in Nürnberg und anderen großen Städten anschlossen, die schon lange nicht mehr gewillt waren, die religiöse und politische Bevormundung durch die geistlichen Stände hinzunehmen. Schon die Renaissance ist nicht nur als kunstgeschichtliche Entwicklung zu begreifen, sondern als neue Kulturepoche, die wesentlich von der wohlhabenden Kaufmannschaft, wie den Medici,

33 Vgl. nochmals Bonhoeffer (1937/2002), Kap. 1 mit dem kritischen Schlagwort von der „billigen Gnade“.
Borgiern u.a. getragen wurde (Burckhard 1860). Das Individuum wurde, wie Küng formuliert, „im Spiegel der Antike (in) seinem Eigenwert als irdische und historische Persönlichkeit" erkannt und von daher „[...] scheint ein freies, selbstverantwortliches, fortschrittliches Menschtum auf" (Küng 2006, S. 545). Renaissance und Humanismus wurden in dieser Hinsicht zusammengesehen und mit dem wesentlichen Anliegen der Reformation als kulturgeschichtlicher Epochenbegriff verbunden. Auch wenn damit der paradigmatische Wechsel nicht auf die Anfänge der florentinischen Frührenaissance (ca. 1420-1500) vorzuverlegen ist, so sind doch die Gemeinsamkeiten der sozialhistorischen Triebkräfte unverkennbar. ${ }^{34}$ Allerdings weiß man auch seit Langem, dass schon zu Lebzeiten des Reformators und erst recht nach dem Tode Luthers heftige Meinungskämpfe ausbrachen und zahllose gemischte Ansichten tradiert wurden, die in ihrer Wirkung auf die Folgezeit mit berücksichtigt werden müssen. Insbesondere scheint die Mitwirkung Melanchthons bei der Abfassung der "Confessio Augustana" aus dem Jahr 1530 und den nachfolgenden Versionen beachtenswert, und das nicht nur wegen der bemerkenswert ökumenischen Ausrichtung, sondern auch weil hier Wurzeln der späteren orthodoxkirchlichen Entwicklung zu sehen sind.

\section{Europäisches Naturrecht der Aufklärung bis Adam Smith und Immanuel Kant}

Schon Franz Wieacker hat treffend bemerkt, dass die lutherische Orthodoxie im 18. Jahrhundert eine Art „Rückkehr [...] [zum] ,thomistischen Aristotelismus' vollzogen" 35 habe; und man ist darin - so wird man ergänzen dürfen - sogar noch ein gutes Stück hinter Melanchthons Humanismus zurückgegangen. Doch ist das nur das Kontrastthema zur folgenden Traditionsgeschichte des kontingenztheoretischen Ansatzes Luthers bei Christian Thomasius.

\subsection{Zur Fortführung des lutherischen Individualismus bei Christian Thomasius}

Im 17. und 18. Jahrhundert setzten sich nach allgemeiner Ansicht die Tendenzen einer „radikal ins Weltliche gekehrten Glaubensüberzeugung“ der Humanität mehr und mehr durch (DiFabio 2005, S. 13). Für die Natur-

34 Vgl. nochmals Küng (2006), Christentum, S. 544 ff.; ausführlich Hansen (2006), S. 43 ff; Schilling, 2017, S.403.

35 Wieacker (1967), S. 264 mit weiteren Nachweisen; Welzel (1962), S. 109. 
rechtslehre wird diese Wende v.a. auf den Niederländer Hugo Grotius zurückgeführt, der sowohl auf Thomasius und Pufendorf als auch auf Christian Wolf erheblichen Einfluss gehabt hat, aber nicht in vollem Umfang rezipiert worden ist. Dennoch wurde der Individualismus der Reformationstheologie und auch - vor allem von Thomasius - der lutherische Glaubensansatz fortgeführt. Wenige Stichworte genügen.

Einer der besten Kenner Thomasius' der Nachkriegszeit, Franz Wieacker, meint zur lutherischen Herkunft, es liege ein „lutherisch-pietistischer Pessimismus" vor, der letztlich auf ockhamistisches Denken zurückgehe (Wieacker 1967, S. 317, Text mit Fn. 19 mit weiteren Nachweisen). Im Anschluss an Ockhams Nominalismus werde nicht mehr an die Grundaussage der älteren Naturrechtstradition angeknüpft, dass der Mensch als Vernunftwesen eine zeitlos gültige Erkenntnis natürlicher Normgrundsätze habe, sondern voluntative Effekte dürften nicht außer Acht gelassen werden. Der menschliche Wille steuere stets die intellektuelle Überzeugtheit des Individuums von normativer Geltung ethischer Grundprinzipien. Dafür habe man auf die berühmte Affektenlehre des Thomasius zu achten, wonach nicht zeitlos gültige Wahrheit naturrechtlicher Normen erkennbar werde, sondern lediglich auf historisch langanhaltende Tradition abzustellen ist. Die Rechtsgeschichte werde zum „Auge“ der Naturwissenschaft, und Thomasius sei in dieser Sicht ein „Begründer des Frühhistoriums“.

Richtig an dieser Einschätzung ist v.a. der Bezug auf die erkenntniskritische Bedeutung der Affektenlehre, die in der Tat grundlegend für das Naturrecht des Thomasius ist. Wegen der Affenktenbestimmtheit menschlicher Erkenntnis gibt es keine zeitlos gültigen moralischen Prinzipien, sondern auch das Naturrecht ist einem gewissen Wandel unterworfen. Thomasius hat dafür sogar eine eigenartige und, wie Wieacker richtig bemerkt, recht modern anmutende Geschichtstheorie entwickelt; denn erstmals in der Entwicklungsgeschichte historischen Denkens wird bei Thomasius in linearer zeitlicher Ereignisabfolge gedacht und damit das zyklische Geschichtsbild der griechischen Klassik überwunden (Hattenhauer1969; Herrmann 1971, S. 102 ff.). Das hat fundierte erkenntniskritische Gründe, die zwar der Überzeugung von der allein seligmachenden Vernunft mancher Denker der Aufklärung widersprechen, aber ganz besonders auf die lutherische Lehre vom verborgenen Gott bezogen sind (Herrmann 1971, S. 79 ff., 88 ff.).

Dennoch hat Thomasius es gewagt, ähnlich wie beinahe zeitgleich auch Locke (Montenbruck 2013,

36 So v.a. Meinecke (1946), S. 28 ff.; zur modernen Lehre eines Naturrechts mit wechselndem Inhalt s.o. zu II.1
S. 297 ff.), eine Art Zivilreligion für den Sinn menschlichen Lebens zu entwickeln, indem er für seine naturrechtlichen Lehren ebenfalls sowohl biblisch als auch vernunftethisch argumentierte. Die wichtigste Besonderheit Thomasius besteht jedoch darin, dass er aus der pietistischen Glaubenstradition heraus argumentiert und dies mit dem aufklärerischen Toleranzdenken verbindet. Dafür wird eine Anthropologie entwickelt, für die die Lehre vom „göttlichen Fünklein“ im Menschen zentral ist (Thomasius 1696/1968, Beschluss Nr. 5, S. 538). ${ }^{37}$

Von Natur aus sei jeder Mensch mit einem Liebesfunken, einer Flamme oder einem Feuer göttlicher Herkunft gesegnet, so dass es schon deshalb nicht nur unchristlich, sondern auch unmoralisch in weltlichem Sinne sei, den Menschen zu quälen, oder inm Gewalt anzutun, um inn zu einer Glaubens- oder Vernunftüberzeugung zu bewegen. Wie Locke in positiv gläubigem Bewusstsein argumentiert (Wieacker 1967, S. 282, 317), so lehnt sich Thomasius an die pietistisch protestantische Glaubensrichtung seiner Zeit an, lässt dabei aber kaum noch substanzmetaphysisches Denken erkennen, sondern bezieht sich ganz auf die Glaubensfunktion der lutherisch-pietistischen Rechtfertigungslehre. Dabei wird durchaus wirtschaftsethisch gedacht, aber die Wirtschaftsethik nicht auf einem Gegensatz göttlichen und weltlichen Glücksstrebens aufgebaut. Denn wenn es dem Einzelnen nicht gut gehe, könne auch die Gesamtheit der Einzelnen nicht vollständiges Glück erfahren; und umgekehrt ist das Glück des Einzelnen nicht vollkommen, wenn es nicht mit dem Glück der Mehrheit im Einklang zu bringen ist, da es um eine „Einheit der Glückseligkeiten“ und den Zusammenhang innerer und äußerer Ruhe des Menschen gehe. Wegen des Liebesfunkens im Menschen strebe dieser nach innerer Ruhe und Mäßigung innerer Affekte, so dass er sich in einer Kunst, das Miteinander der Menschen zugleich vernünftig und tugendhaft zu gestalten, üben müsse (Thomasius, 1696/1968 ${ }^{38}$ ). Dafür werde aber gleichermaßen eine quietas externa vorausgesetzt, die vom Glück der Mehrheit anderer Menschen abhängig sei (Thomasius 1720/1963, I. Buch IV. Cap. § 37, dt. Ausgabe S. 130 f.). ${ }^{39}$ Damit wird deutlich, dass es für

37 Zur Entsprechung bei den Pietisten Arnold, Spener u.a. vgl. Herrmann (1971), S. 73.

38 So der Titel des Buches Von der Kunst Vernünftig und Tugendhaft zu lieben, Als dem einzigen Mittel zu einem glückseligen, galanten und vergnügten Leben zu gelangen; oder Einleitung der Sitten-Lehre, 8. Aufl., 1726.

39 Lateinischer Titel : Fundamenta Juris Naturae Et Gentium Ex Sensu Communi Deducta ; ergänzend heißt es an der zitierten Stelle: „Man muss aber dieses Gute einander nicht entgegensetzen oder voneinander absondern, als wenn das ehrbare Gut der Wesen nicht nützlich oder angenehm wäre"; dazu Herrmann (1971), S. 91 f.; zur Entsprechung mit utilitaristischen Lehren siehe Horn (2007), S. 52. 
Thomasius, ganz wie im englischen Sensualismus, entscheidend auf die gefühlsmäßige Erfahrung des Menschen ankommt. Seine Variante der Zivilreligion ist also von der Warte des pietistisch-mystischen Gläubigen aus entwickelt und dennoch, ja gerade deshalb dem Empirismus eines John Locke eng verbunden.

Dass hier und in Christian Wolffs Hauptwerk „Institutiones juris naturae et gentium“ von 1750 (1754, § 194) bereits lange Zeit vor Adam Smith eine Art Marktwirtschaftstheorie „im Gewande der Ethik“ vorformuliert worden ist, gehört zu den neueren Entdeckungen der Wirtschaftsrechtsethik (Horn 2007, S. 55). Doch ist die Entwicklung, wie gezeigt, schon im Humanismus und in der Frührenaissance vorgeprägt und noch deutlich weitergehend bereits hier und nicht erst in der Aufklärung anthropologisch ausgerichtet. Übereinstimmende Grundlage scheint eine positivere Einschätzung des Weltlichen und Naturhaften zu sein (Herrmann 1971, S. 131 passim), die nicht mehr substanz-metaphysisch auf Gegensätze von Ideen und Wirklichkeit oder auf der Vorstellung der Sündhaftigkeit der Welt aufgebaut ist, sondern den Menschen als vernunftbegabt und mit „Fünklein“ von Liebesaffekten ausgestattet sieht.

\subsection{Hobbes' animalistische Anthropologie}

Besonders scharf entgegengesetzt wirkte die wenngleich ebenfalls gänzlich empiristisch verstandene - Lehre von Hobbes, der wohl der Erste war, der das Wesen des Menschen in einer raubtierhaften Gier entdeckte. Es ist, als hätte Hobbes nicht nur die schrecklichen Machtkämpfe im zerrissenen England seiner Zeit im Auge gehabt, sondern auch bereits die rüden Missbräuche von Investmentbankern auf den Kapitalmärkten zu Beginn des 21. Jahrhunderts vorhergesehen. Grundlage dafür war seine Erkenntniskritik (Sensualismus).

Fragt man, ob auch insofern eine Erkenntniskritik zugrunde liegt, die man als Lehre des Nichtwissens bezeichnen kann, so führt die Metaphysikfeindlichkeit in die Irre; denn Hobbes wähnte sich in sehr genauem Wissen darüber, dass der Mensch vom Selbsterhaltungstrieb bestimmt sei, und dass als Maßstäbe des Handelns demzufolge v.a. der Nutzen (utilitas/utility) und der Egoismus des Menschen in Betracht kommen. Das führte zu der ebenfalls keineswegs bloß sozialempirisch begründeten Vorstellung, dass die Menschen ohne einen Staatsvertrag mit einem mächtigen Herrscher einander teuflisch ausbeuten und umbringen würden $(\text { Leviathan })^{40}$. Wie schon bemerkt, scheint ein Rück-

40 Thomas Hobbes, Leviatan (1651/1991), Kap. 13 zur Lit. s. Horn (2016), Rdn. 315 f. fall in mittelalterliche Herrschafts- und Denkstrukturen vorzuliegen (Wieacker 1967, S. 264).

\subsection{Adam Smith - Ein Menschenbild mit reformationstheologischem Hintergrund}

Die Wettbewerbslehre des Adam Smith ist oft als Anlehnung an Hobbes' Raubtieranthropologie missverstanden worden. Berühmt ist die Einschätzung von Karl Marx, Smith sei ein guter Freund von Hobbes und, wie dieser, ein strenger Atheist gewesen. ${ }^{41}$ Noch in neuerer Zeit nimmt der Chicago-Ökonom Stigler an, das Wettbewerbskonzept sei aus dem darwinistischen Modell des Überlebenskampfes herzuleiten, das mit den Hobbes'schen Lehren bestens zusammenpasst. ${ }^{42}$ Nach anderer Ansicht weicht Smith gerade im Menschenbild entscheidend von Hobbes ab und tritt in der Auseinandersetzung mit Mandelville zur Bienenparabel geradezu für eine weitgehende Moralisierung des Wettbewerbs ein. ${ }^{43}$ Teils geht man sogar so weit anzunehmen, dass Smith allein wegen des Gegensatzes zu Hobbes und wegen der später so umstrittenen gesellschaftlichen Wettbewerbsfunktionen ${ }^{44}$ sein damals neues ökonomisches Modell entwickelt hat. Lachmann meint: „Nur weil Adam Smith dieser Einsicht vertraute, konnte er für freie Märkte eintreten." (Lachmann 1989, S. 101). Ähnlich aber weniger weitgehend, wird im Vergleich zu Hobbes eine gewisse „Sublimierung des Vorteilsbegriffs" gesehen. ${ }^{45}$ Auch haben Lachmann ${ }^{46}$ und unlängst wieder Sedlacek betont, dass die viel beachtete Sympathievorstellung bei Smith (Lachmann 1989, S. 100) in einem besonderem Gegensatz zu Hobbes' und Darwins Grundannahme vom unsozialen Vernichtungskampf steht: Danach ist der Mensch bei Smith nicht nur ein soziales Wesen, sondern seine Natur ist in dem Bedürfnis verwurzelt, Empathie zu empfinden. Es geht also keineswegs nur um Überlebensstrategien im struggel for life wie Hobbes und Darwin es sahen, sondern um Sympathie und vielleicht sogar schon Empathie im Sinne gesteigerter Anteil-

41 Marx (1867/1968), S. 645 f.: Atheismus; für „Neo-Epikureer“.

42 Stigler (1975), S. 30 ff.: Survival oft the Fittest, zitiert nach Schmidt (1999), S. 20.

43 Vgl. Meyer (2014), S. 399 f.; näher s.u.

44 Meinungsstand bei Schmidt (1999), S. $5 \mathrm{ff}$.

45 So Horn (2007); dazu Smith (1759/1994), S. 506: „Die Rücksicht auf unser eigenes Glück [...]erscheint [...] als lobendes Prinzip des Handelns. Charaktergewohnheiten wie Wirtschaftlichkeit, Fleiß, Umsicht, Aufmerksamkeit, geistige Regsamkeit, werden nach allgemeinem Dafürhalten aus eigennützigen Beweggründen gepflegt, und doch hält man sie zugleich für sehr lobenswürdige Eigenschaften [...]"; dazu auch Meyer (2014), S. 399 f.

46 Lachmann (1989), S. 100; zur Abtrennung der Ethik von den Wirtschaftswissenschaften seit Bodinus im 16. Jahrhundert s. Ebd., S. 34 unter Hinweis auf Müller-Armack (1970), S.19 ff. 
nahme sind gemeint. Soviel zur heute wohl überwiegenden Ansicht. ${ }^{47}$

Sehr umstritten war des Weiteren das Verhältnis des Schotten Adam Smith zur deutschen und französischen Aufklärung. Teils nahm man an, es bestehe geradezu ein Gegensatz, da Rousseau und Voltaire letztlich auf die natürliche Gleichheit aller Individuen abgezielt hätten, während es Smith gerade um die individuelle Freiheit gegenüber dem Staat und nicht um Gleichheit gegangen sei. ${ }^{48}$ Die Gegenansichten gehen zum einen dahin, Smith folge einer wesentlich deistischen Vorstellung prästabilisierter Harmonie, wie sie v.a. Leibnitz ausgearbeitet hat (Vogl 2011; Lachmann 1989, S. 102). Denn die Annahme eines Preisgleichgewichts unter Bedingungen atomistischen Wettbewerbs sei von einem Harmoniedenken geprägt, das im göttlichen Schöpfungsakt als Prästabilisierung begründet sei.

Zum anderen nimmt man, wie schon oben (zu V.1) erwähnt, neuerdings an, dass sehr viel engere Zusammenhänge zwischen Smith und der französischen und deutschen Aufklärung bestanden haben, und dass dies gerade in den freiheitlichen Lehren vom individuellen Lebensglück erkennen sei. Wie Smith die Auswirkungen des Glücksstrebens Einzelner auf die gesamtwirtschaftliche Wohlfahrt zur Grundlage seiner Wettbewerbslehre gemacht habe, so sei bei Thomasius u.a. das schon erwähnte Zusammenspiel von Gemeinwohl und individueller Seelenruhe betont (Horn 2007, S. 52 m.w.N.). Hier wird im Wesentlichen der zuletzt genannten Ansicht gefolgt, zumal für diese zahlreiche weitere Belege zur Weiterführung gesamteuropäischer Rechtstraditionen in der englischen und schottischen Aufklärung angeführt werden. Auch dürfte das bekannte Zusammenspiel von Freiheit, Gleichheit und Brüderlichkeit nicht erst in der französischen Revolution maßgebend geworden, sondern schon von Rousseau und Voltaire vorbereitet worden sein. Ein zwingender Gegensatz von Gleichheits- und Freiheitsaspekten schien dem deutschen Aufklärer Thomasius keineswegs gegeben, sondern im Rahmen der erläuterten Glückslehre überwunden (s.o. zu V.1).

Andererseits ging die Verbindung des Adam Smith zu deutschen Aufklärungsphilosophen denn wohl doch nicht so weit, dass er auch die Leibnitz'sche Harmonievorstellung übernommen hätte. Denn die Hand-Theologie des Alten Testaments und deren sogleich noch näher belegte Berücksichtigung bei Smith ist kaum mit einer deistischen Gottesvorstellung vereinbar. Der Gott Davids greift nach 1. Sam. 24, 13 f., 26, 23

47 Zum älteren Meinungsstreit vgl. Todt (1991) S. 1, 3

48 So v. Hayek (1967), S. 11 ff.; zustimmend auch Schmidt (1999), S. 2. etc. energisch in die Geschichte ein und bewahrt Israel vor der erneuten Machtübernahme des Saul'schen Herrscherhauses. Dem entsprechend stellt sich auch bei Smith das Preisgleichgewicht nicht von selbst ein, sondern es bedarf insbes. der Einsicht des staatlichen Souveräns, der bisher überwiegend für richtig gehaltene Konzeption des Merkantilismus nicht zu folgen und Marktregulierungen weitestgehend zu unterlassen. Zudem gibt es sogar Textnachweise im Werk von Smith, wonach kartellmäßige Absprachen von Marktteilnehmern wettbewerbsschädlich seien. Solches Gotteshandeln und mögliche Gleichgewichtsstörungen sind bei prästabiler Schöpfung nicht vorstellbar. ${ }^{49}$

Sedlacek hat nicht zuerst, aber mit den umfänglichsten Belegen darauf aufmerksam gemacht, dass die Handvorstellung zutiefst religiöse Wurzeln hat, die v.a. auf die Gnadenlehre des Alten Testaments zurückgehen. Doch wird weder bei ihm selbst noch bei seinen Kritikern hinreichend erkannt, dass damit zugleich an die protestantische Rechtfertigungslehre und den Paradigmenwechsel des Individualismus der Neuzeit angeknüpft wird.

Immerhin wird Smith's Metaphysikkritik herangezogen, die jedenfalls, wie erläutert, eine wesentliche Voraussetzung des neuen Menschenbildes war. Denn wie man das Wesen der Dinge nicht metaphysisch erklären könne, so sei auch nicht kausal zu definieren, weshalb ausgerechnet der Egoismus Einzelner im Wettbewerbsmarkt zum Wohle Aller im Sinne eines altruistischen gemeinen Wohls bestmöglich beitrage. ${ }^{50}$ Aber gerade wegen dieses Nichtwissens bezeichnet Smith den Wettbewerbsmechanismus als unsichtbar und denkt dabei an eine imaginäre „Hand“. Wer sich in die Literatur dieser Zeit eingelesen hat, erkennt sofort, dass damit auf die Glaubenstradition der gnädigen Handreichung Gottes angespielt ist ${ }^{51}$ und letztlich sogar über die biblische Tradition hinausgehende bis zu mesopotanischen, ägyptischen, griechischen und lateinischen Überlieferungen reichenden Erzählungen über unsichtbare Gotteshände verwiesen werden soll. Man wird diese Absicht als Grund dafür anzusehen haben, dass

49 In diesem Sinne wird man auch den Streit zur Deutung der berühmten Bienenparabel von Hutchison und deren Verhältnis zur Glückslehre des Adam Smith zu beurteilen haben. Doch muss dazu hauptsächlich auf die Literatur und auf Herrmann (2018) verwiesen werden (ebenso zur Hand-Theologie des Alten Testaments).

50 Zur Leugnung kausaler Gesetzmäßigkeit als Prinzip theoretischer Vernunft schon Hume (2005), sec.2 § 5; zum Gegensatz zu Kant s. ders. (1788), Bd. IV, S. 15 ff.; dazu Horn (2016), Rdn. 326.

51 Vgl. Sedlácek (2013), S. 321 ff., 322 mit Zitat von John Boli (1995), S. 93, 97: „Die unsichtbare Hand ist ein mystischer Gott, der auf geheimnisvolle [...] Weise [...] holistische Vorteilhaftigkeit hervorbringt, die sich aus den unheiligen Motivationen der von Eigeninteressen erfüllten Akteuren nicht vorhersagen lässt; vgl. auch R. Precht (2017), S.360. 
Smith gelegentlich einfach von der „unsichtbaren Hand Jupiters" spricht, um den theologischen Hintergrund wenngleich mit außerchristlichen Unterton anzudeuten.

Hält man sich diesen Traditionszusammenhang vor Augen, so wird klar, warum Smith seine Wettbewerbslehre in einem derartigen Widerspruch von Egoismus und "unsichtbarer Hand" inszenierte. Zwar reicht der Egoismus schon wegen seiner Sublimierung bei Weitem nicht an den Unrechtsgehalt des biblischen Intriganten David heran. Aber die Gnadenvorstellung beruht offenbar nicht auf einer wiedergutmachenden Ablassleistung oder sonstiger Leistungsgerechtigkeit, sondern auf bloß gnädiger Handauflegung Gottes. Luthers Lehre von der sola gratia scheint aus dieser Sicht zwar immer noch ziemlich weit entfernt, da im Alten Testament natürlich noch nicht von einer Versöhnung durch Christus die Rede ist. Aber es geht doch um eine sündenvergebende Segnung. Nur ist bei Smith eben nicht Luther, sondern die alttestamentliche Tradition der unsichtbaren Hand in Bezug genommen. Inwieweit mittelbar der Bezug vorliegt, weil auch Luther sich natürlich mit Davids Segnungen nicht nur in der Bibelübersetzung, sondern auch in seinen Predigten befasst hat, muss hier ebenso offen bleiben wie Näheres zur Entmythologisierung. ${ }^{52}$

\subsection{Kantische Utilitarismuskritik und Kontingenzlehre}

Trotz des bisherigen Befundes möchte man solange nicht von einem Paradigmenwechsel sprechen, wie der weitere Verlauf der Aufklärung bis Kant und dessen anthropologischen Grundannahmen nicht gegenübergestellt sind. ${ }^{53}$ Das kann hier aus Raumgründen nicht vollständig geleistet werden. ${ }^{54}$ Nur Folgendes soll hervorgehoben werden.

a. Rationalität und autonome Persönlichkeitsentfaltung. Kant bestreitet jegliche Vernunftmäßigkeit des Glaubens, da die Vernunft des Menschen der Offenbarung nicht untergeordnet sei (Troeltsch 1913, S. 373). Jenseitiges Sein und zu allererst auch das Sein einer Gottheit sei nicht durch Denkschlüsse, wie den der logischen Notwendigkeit des unbewegten Bewegers erweislich, da jegliches Allmachtsdenken zugleich voraussetzt, dass es jenseits der Denkbarkeit besteht. Gottesbeweise seien deshalb in sich widersprüchlich und stellen eine Anmaßung menschlichen Wissens

52 S. aber die Nachweise in Herrmann (2018).

53 Zur Ansicht Kants, inm selbst sei ein paradigmatischer Wechsel zuzuschreiben, vgl. Kant (1788), Bd. IV, Vorrede zu Bd. XI.

54 Näher Schelika (2007); dazu Herrmann (2018). dar. ${ }^{55}$ Mit diesem Argument der Wissensanmaßung stellt er sich, wenngleich nicht biblisch vertiefend ${ }^{56}-$ in die Tradition der Erkenntniskritik, die bei der Paradiesgeschichte bis hin zum Sensualismus von Hobbes und dem Verdikt wettbewerblicher Wissensarroganz bei Adam Smith reicht. ${ }^{57}$

Vollkommen treffend ist damit auch eine Spekulationskritik gegenüber naturrechtlichen Erkenntnissen wissenschaftlicher Art verbunden, da die Kenntnis der Natur auf Sinneserfahrung beruhe, also a posteriorisch sei, während moralisches Handeln auf eine im Innern des Menschen vorhandene Vorstellung möglichen gesetzmäßigen Lebenssinns zurückgehe und demzufolge als a priorischer Vernunftschluss eingeordnet wird. ${ }^{58}$ Kant bestimmt diese selbst gesetzte Prämisse als eine, die allgemeine Anerkennung verdienen soll (kategorischer Imperativ): „Handle so, dass die Maxime deines Willens jederzeit zugleich als Prinzip einer allgemeinen Gesetzgebung gelten könne." (Kant 1788 zu A54). In krassem Gegensatz zum Utilitarismus formuliert Kant einen konsequenten Altruismus, wonach jegliches Motiv des Eigennutzes dazu führt, dass die Handlung nicht moralisch gerechtfertigt werden kann. Diesbezüglich sind die meisten Anhänger der Ignoranzlehre zu Recht anderer Ansicht. ${ }^{59}$ Denn mit dem Wissen um das Gute des Altruismus setzt auch Kant nicht weniger als die meisten Naturrechtler (Finnis 2011, S. 97) dem Vorwurf unzulässig angemaßter Letztwahrheit aus, anstatt sich mit der bloßen Möglichkeit seines altruistischen Lebenssinns zu bescheiden. ${ }^{60}$ Diese nicht streng durchgehaltene Kritik der Wissensanmaßung metaphysischen Denkens teilt Kant aber durchaus mit frühen Thomas-Kritikern, wie Duns Scotus und Ockham. ${ }^{61}$

Wichtiger ist deshalb der Gedanke der Autonomie der Persönlichkeit, den er der o.a. Vorstellung von

55 Vgl. nur Vorrede (1788), D XIX, Akademieausgabe (1968), Bd. IV; dazu Hailer (2014), S. 33 f.

56 Anspielung auf den Turmbau zu Babel aber bei Kant (1788) zu A 707/B 735.

57 Wohl aber ist die Vorsehungslehre der Bibeltradition und immerhin auch die Geschichte vom Turmbau zu Babel kritisch behandelt, Kant (1788), A 707/B735; näher Schelika (2007), S. 375.

58 Vgl. auch Horn (2016), Rdn. 330.

59 Besonders deutlich der Sensualist Hobbes, s.o. zu IV.2, aber auch Erasmus (a.a.O. zu IV.1) kennt keine generelle Egoismuskritik; anders Plato, s. Herrmann (2017).

60 Zutreffend deshalb schon der Spott von v. Goethe und Schiller (1797): „Gern dien ich den Freunden, doch tu ich es leider mit Neigung [...]; zu Widersprüchen des kantischen Subjektivismus vgl. vor allem Whithead (1963/1985), S. 85; dazu Deuser (2009), S. $311 \mathrm{f}$.

$61 \mathrm{Vgl}$. nur den Hinweis auf Ockham - und übrigens auch auf Plato bei Horn (2016), Rdn. 337; näher zu Platos Wissensbegrenztheit im Höhlengleichnis Herrmann (2017) zu III.2; zu Duns Scotus und Ockham Ebd. zu III.3. 
Hobbes entgegensetzt, der Herrscher sei zur Rechtssetzung berufen, um den animalischen Kannibalismus des Menschen zu beherrschen (s.o. zu IV.2). Kant nimmt in gewisser Weise das Umgekehrte an: nicht der Souverän ist Quelle des Rechts, sondern das Recht ist Ursprung und Rechtfertigung staatlicher Herrschaft. ${ }^{62}$ Die Bürger sind im Staat nicht nur dem Recht unterworfen, sondern sie wirken zugleich als Gesetzgeber, indem sie durch unveräußerliche Rechte, wie v.a. die Meinungsfreiheit, zur öffentlichen Kritik an Akten der Staatsgewalt berechtigt und aufgerufen sind. ${ }^{63}$ Damit nähert sich Kant bereits dem heutigen Konzept demokratischer Öffentlichkeit an (Mestmäcker 1994, S. 62 f.). Zunächst aber verweist Kant darauf, dass diese Öffentlichkeitsfunktion nur erfüllt werden kann, wenn das Individuum darauf achtet, frei von Machteinflüssen anderer Menschen und des Staates zu sein. Als einziges Naturrecht des Einzelnen erkennt Kant (1797, S. 230 f.) die Freiheit im Sinne von Unabhängigkeit von der Willkür anderer Menschen. Staatliches Recht ist zwar Zwangsordnung, muss also befolgt werden. Es darf und soll aber vom Bürger kritisch beurteilt werden, um für die Zukunft für eventuelle Abänderungen zu sorgen. Das gehört grundlegend zur Autonomie der Persönlichkeit i.S. Kants (Mestmäcker 1994, S. 63).

Mit der autonomen Freiheit der Persönlichkeit wird zugleich ein wichtigerAspekt zur wettbewerblichen Handlungsfreiheit berührt, den Kant allerdings selbst nicht ausdrücklich behandelt. Denn wie öffentliche Kritik am Souverän staatsbegründend ist, so kann die freie Entscheidung im Wirtschaftsverkehr das allgemeine Wohl befördern, indem v.a. das Preisgleichgewicht herbeigeführt wird, das sowohl für Mäßigung des Anbieters und Nachfragers als auch dafür sorgt, dass die Nachfrage auf denjenigen Anbieter gelenkt wird, der zu niedrigsten Kosten produzieren kann. Allerdings sind es in dieser Sicht nicht die ökonomischen Gemeinwohlvorteile, die den Wettbewerb ethisch wertvoll erscheinen lassen, sondern die Beförderung der Autonomie der Persönlichkeit; denn ebendieser Autonomiegedanke ist für die kantische Ethik zentral.

Kritisch lässt sich einwenden, dass mit dem Autonomieprinzip entweder die Möglichkeit impliziert wird, dass der Einzelmensch die für inn geltenden Maßstäbe selbst beliebig festsetzen kann; oder dass, wie Kant meint, von einer Allgemeingültigkeit des Sittengesetzes auszugehen ist. Ersteres ist offenbar sinnwidrig; Letzteres scheint aber auch nicht unproblematisch, weil die allgemeine Gültigkeit ein gewisses Maß an Heteronomie für jeden einzelnen Betroffenen notwendig

62 Dazu und zu Folgendem. s. Mestmäcker (1994), S. 55, 59 ff. 63 Kant (1793), S. 303. zur Folge hat. ${ }^{64}$ Kant scheint also insofern ebenso zu rigoristisch, wie er es im Hinblick auf die Utilitarismuskritik des kategorischen Imperativs nach hier vertretener Ansicht ist.

b. Kontingenter Lebenssinn. Ein weiterer primär erkenntnistheoretischer Ansatz Kants ist neben dem kategorischen Imperativ lange Zeit weitgehend unbeachtet geblieben: die Transzendentalphilosophie. ${ }^{65}$ Erst die neueren Religions- ${ }^{66}$ und Orientierungsphilosophien $^{67}$ haben den Gedanken wieder aufgegriffen und wesentlich weiter entwickelt. Doch zunächst zum a priorischen Denkmodell von Kant selbst, ${ }^{68}$ das im Ausgangspunkt vollständig an seine metaphysische Skepsis anschließt: Während der kategorische Imperativ in der Kritik der praktischen Vernunft begründet wurde, ist die Transzendentalphilosophie in der Schrift zur theoretischen Vernunft zu finden. Danach ist zunächst mit dem Verstehen von Sein notwendig die Bedingung der Möglichkeit von Erkenntnis verbunden. D.h. die „reine Vernunft" muss mit der Suche nach etwas Wahrem, das ist und als solches auffindbar vorhanden ist, zwingend voraussetzen, dass sich die Erkenntnis irgendwie erfolgreich auf eine Wirklichkeit bezieht, die ist und nicht vielmehr nicht ist. Zwar könne man das nicht überprüfen, wohl aber habe die Vernunft es als Bedingung der Möglichkeit des Erkennens anzuerkennen (Kant 1788, zu B 672). Dadurch wird alles Sein möglich (kontingent), auch wenn es dem Menschen nicht gegeben ist, die Wirklichkeit ebendieses Seins objektiv zu erkennen und darüber eine beschreibende Theorie zu entwickeln. Der Mensch muss mit dem Erkennen als Solchen subjektiv setzen, dass ein Objekt des Erkennens möglich ist. Kant versteht dies als das Transzendentale seiner Erkenntnistheorie und spricht insofern auch von einer "regulativen Idee“, die man auch Gott nennen könne, wenn man nur darauf achte, dass daraus keine Theorie über ein Sein Gottes werde. ${ }^{69}$

Ebenso müsste es eigentlich auch mit der praktischen Vernunft sein. Denn mit der Vorstellung einer von der Vernunft gebotenen Pflicht, die dem Handeln aus egoistischer Neigung widerspricht, ist denknotwendig die Möglichkeit verbunden, dass eine Welt entsteht,

64 Vgl. nur Horn (2016), Rdn. 333, m. Verweis auf weitere heteronome Implikationen bei Annahme göttlichen Ursprungs des allgemeinen Sittengesetzes.

65 Zum Gottesbegriff als „regulative Idee“, die die Vernunft zu setzen habe vgl. vor allem Kant (1787), zu B 672 u.ö.; dazu Hailer (2014), S.35

66 Vgl. grundlegend Rentsch (2000), S: 58 ff., 67 ff., 72 ff.; zusammenfassend (Hailer) 2014, S. $48 \mathrm{ff}$.

$67 \mathrm{Vgl}$. zusammenfassend zunächst Stegmaier (2008).

68 Zum Folgenden s. Kant (1787), zu B 672 u.ö.; zusammenfassend Hailer (2014), S. $35 \mathrm{ff}$.

69 Dazu in religionsphilosophischer Hinsicht Hailer (2014), S. 35 m.w.Nachw. 
die dem Leben aus Pflicht entspricht. ${ }^{70}$ Aber hier bleibt Kant nicht beim Kontingenzdenken seiner eigenen Lehre von der bloßen Möglichkeit, die auch auf der Ebene der Pflichtenethik bei kontingentem Möglichsein bleibt, sondern nimmt an, die einzig akzeptable Ethik sei die aus Vernunft und aus dem, was die Vernunft gebiete (vernünftige Pflichterfüllung). Was folgt, ist die Moralität des Pflichtbewusstseins, die nach zutreffender Ansicht eine Entgleisung in objektive Aussagen über das Gebotensein bestimmter Pflichtinhalte darstellt (Horn 2016, S. 337). Zum einen hat das in der Folgezeit nicht ohne Grund zu maßlosen Übertreibung geführt (Befehlsgehorsam und „Befehl ist Befehl“, s. Horn 2016, Rdn. 340); und zum anderen ist mit dem Kategorischen der Kantethik ohne zwingende Logik der Gedanke der Gleichbehandlung verbunden. Darauf hat später Rawls verwiesen und gemeint, man müsse aus dem Gedanken der Unerkennbarkeit inhaltlichen Lebenssinns (veil of ignorance) einen Vorrang des Interesses des jeweils am schlechtesten Gestellten herleiten (sog. Differenzprinzip, Rawls 1971, S. 95 ff., 115 ff.). Darauf und auf die auch insofern angebrachte Kritik wird sogleich zurückzukommen sein. Im Hinblick auf die Kontingenzlehre ist vorerst nur festzustellen, dass der Gleichheitssatz und mit diesem zugleich natürlich auch das konsequent altruistische Prinzip - nicht mehr im Rahmen dessen bleibt, was aus der bloßen Bedingung der Möglichkeit von Lebenssinn folgt.

Kant hat den transzendentalphilosophischen Ansatz für seine Ethiklehre wohl auch deshalb nicht konsequent zu Ende gedacht, weil inm der Gedanke noch fremd war, dass allein aus der Möglichkeit von Lebenssinn tiefgreifende ethische Folgerungen herzuleiten sind. Zu solchen Einsichten kam man erst in neuerer Zeit in der Folge von Thomas Rentschs neo-kantianischen Lehren $^{71}$ und deren Fortführungen der neueren Religionsphilosophie. ${ }^{72}$ Das wirklich Weiterführende liegt nicht im kategorischen Imperativ und der damit verbundenen aber nicht ganz widerspruchsfrei begründeten - Lehre von der Autonomie der Persönlichkeit, sondern in der Kontingenzlehre.

Kant sieht, soweit ersichtlich, erstmals, dass Wesensund Sinnerkenntnis nicht nur nicht metaphysisch begründet werden kann. Das haben Andere bereits lange vor inm erkannt. Aber er analysiert als Bedingung der Möglichkeit von wahrem Sein und von Lebenssinn die von ihm sog. regulative Idee. Das ist ohne Vorbild in der Geschichte des Individualismus und hat zu einer ganzen Reihe von neuen Lehrmeinungen geführt, von denen hier

70 Kant (1787), A 226; dazu Hailer (2014), S. 36

71 Rentsch (2000); ders. (2005), S. 124 ff.

72 Dazu nochmals dazu Hailer (2014), S. 36. nur auf die sprachanalytisch-religionsphilosophische von Wittgenstein und Rentsch hinzuweisen ist. ${ }^{73}$ Indessen ist die konsequente Durchführung der Kontingenzlehre nicht bei Kant selbst gelungen, sondern erst im Zusammenhang mit der negativen Theologie entwickelt worden (Hailer 2014, S. 48 ff.).

Damit wird die Untersuchung der historischen Ursprünge wirtschafts- und rechtsethischer Humanitätsvorstellungen abgeschlossen. Das geschieht nicht in der Annahme, dass nach Smith und Kant keine wesentlichen Beiträge zum Individualismus und Subjektivismus oder zur Freiheitsethik und zur Kultur der Freiheit mehr geleistet wurden. Doch gehört das nicht mehr zur Entstehung des Paradigmas individualistischer Lehren, sondern zur sog. normalen Wissenschaft i.S. des Kleinarbeitens und Verfeinerns. Denn an eine Überwindung des Individualismus als Grundelement des Denkens und Handelns ist weder in der neueren philosophischen Literatur ${ }^{74}$ noch in der soziologischen, ökonomischen und rechtsethischen Lehre ernsthaft gedacht. Dafür muss auf die Literatur verwiesen werden. ${ }^{75}$ Für die Geschichte des normativen Individualismus und dessen wettbewerbsethische Implikationen ist der reformatorisch-kontingenztheoretische Ansatz entscheidend, der bis zu Kant nicht nur beibehalten, sondern philosophisch weiterentwickelt und präzisiert worden ist. Es sei lediglich darauf hingewiesen, dass neuere Lehren des sog. Kommunitarismus versuchen, zu starke Vereinseitigungen des normativen Individualismus aufzubrechen. ${ }^{76}$ Paradigmatischer Wandel scheint sich darin aber nicht anzudeuten. Das Folgende ist deshalb auf transzendentalphilosophische Lehren konzentriert, die auf den kantischen Kontingenzansatz zurückgehen.

73 Rentsch (2005), S. 124 ff.; zu weiteren Auswirkungen der Kontingenzlehre auf Hegel, Kierkegaard, Pierce und Benjamin, Ebd., S. 139 ff.; zur Rezeption bei Wittgenstein, Ebd., S. $158 \mathrm{ff}$.

74 Vgl. die Nachw. o. Fn. 2; zum Anschluss der modernen Rechtsethik an den Idealismus von Hegel und Fichte, aber auch zu fernöstlichen Lehren neustens Zaczyk, Selbstsein und Recht (2014) m.w.Nachw., insbes. S. $31 \mathrm{ff} ., 44 \mathrm{ff}$

75 Vgl. besonders Luce (2017), S. 29 u.ö.; dazu schon o. I vor 1

76 Vgl. nur von der Pfordten (2011), S. 283 ff. mit w. Nachw.; zusammenfassend ebd. S. 462: „Eine gemäßigte Form des Kommunitarismus verlässt die normativ-individualistische Theoriegruppe nicht"; zu Ansätzen der phänomenologischen Naturrechtslehre vgl. nur Horn, (2015), S. 99 f. mit w. Nachw.; zu den Bezügen phänomenologischer Erkenntniskritik auf theologische Glaubenslehren Boehnke (2013), S. 451, 456: "Idealismus der Phänomenologie Husserls“; zur transzendentalphilosophischen Einordnung Husserls vgl. Stegmaier (2008), S. 119; Der kulturwissenschaftliche Ansatz von Zaczyk (2014) bei Fichte ist beachtlich, aber ebenfalls nicht paradigmentheoretisch als Umbruch einzuordnen. 


\section{Folgerungen zur Steuerungsethik der Gegenwart}

\subsection{Zur amerikanischen Kontingenzethik seit Rawls}

Vor allem die Auswirkungen auf die amerikanische Transzendentalphilosophie und speziell auf Rawls bereits erwähnte Lehre vom Schleier des Nichtwissens (veil of ignorance) sind hervorzuheben. Denn damit ist nicht nur die kantische Meta-Ethik weitergeführt, sondern es sind auch deren protestantische Ursprünge seit der Kontingenztheologie der lutherischen Rechtfertigungslehre fortentwickelt. Man braucht kein Rawls-Schüler zu sein, um zu erkennen, dass es eines seiner wichtigsten Anliegen war, die Ignoranzvorstellung der kantischen Ethik konsequenter durchzuhalten, als es Kant selbst gelungen war. An die Stelle des kategorischen Prinzips des konsequenten Altruismus trat der Schutz Schwächster im Sinne des sogenannten Differenzprinzips, d.h. bei der Untersuchung der Folgen einer Entscheidung sollte stets diejenige Alternative den Vorzug verdienen, die etwaige Nachteile für den am schlechtesten Gestellten in der relevanten Gemeinschaft minimiert, bzw. die Vorteile für diesen möglichst verbessert (Rawls 1971, S. 95 ff., 115 ff.).

Allerdings ging auch dieser Versuch aus verschiedenen Gründen nicht auf. Besonders intensiv kritisiert worden ist die fehlende Unterscheidung von mehr oder weniger gemeinschaftsrelevanten Interessen im Sinne kommunitarischer Konzepte und von Werten der Menschenwürde, Persönlichkeitsautonomie etc. (von der Pfordten 2011, S. 422 ff., 428 ff.). Dennoch ist der Rawls'sche Ansatz wiederum unter Aspekten der Kontingenz weiterentwickelt worden, indem für die Beurteilung der Schutzwürdigkeit von Interessen Schwächster abgestellt wurde, ob der Schutz aus Gründen der Leistungsbereitschaft verdient erscheint. Dazu hat unlängst Mounk darauf hingewiesen, dass es im Sozialrecht der USA seit Langem eine Rechtsprechung gibt, die für die Zuerkennung staatlicher Sozialhilfen bei Arbeitslosigkeit etc. auf die Arbeitswilligkeit des Empfängers abstellt (Mounk 2017, S. 26 ff., passim). Mit Bezug auf Rawls „account of natural and social contingencies" (Rawls 1971, S. 247) sei dieser Ansatz zwar als eine Art VerdienstRechtfertigung (merit) unakzeptabel (Rawls 1971, S. 107), aber doch dahin korrigierbar, dass statt des Erfolgs- und Leistungsverdienstes ein bestimmtes Kontrollprinzip von Verantwortungsbereitschaft (Control Principle/accountability) zugrunde gelegt werde (Rawls 1971, S. 111 mit weiteren Nachweisen). Mit
Strawson (1986/1994, S. 13 f.) wird der seit Beginn des „Zeitalters“ ungelöste Meinungsstreit zur Willensfreiheit (age-old debate on free will) dahingestellt, dafür aber vorausgesetzt, dass wahre Verantwortlichkeit im Sinne eines gewissen moralischen Respekts (certain crucial moral respect) feststellbar sein müsse (Ebd., S. 119).

Konkrete Einzelheiten dazu bleiben sowohl bei Strawson als auch bei Mounk auf den Bereich des Sozialrechts fixiert, so dass verallgemeinernde Aspekte der Verantwortungsbereitschaft wettbewerblicher Handlungsfreiheit bislang nicht ausgearbeitet sind. ${ }^{77}$ Aber es gibt doch Anhaltspunkte zumindest insofern, als an die Stelle von leistungsgerechter responsibility ein Verantwortungsprinzip im Sinne von accountability treten soll. Der erforderte moralische Respekt des schützenswerten Marktteilnehmers betrifft nämlich im Kern den Nachweis, dass eine gewisse Risikovorsorge getroffen worden ist. Alternativ wird man jedenfalls zu verlangen haben, dass Rücksichtnahme im Sinne eines objektiv nachvollziehbaren risk management vorliegt.

\subsection{Aktuelle Fragen der Ethik privatrechtlicher Steuerung}

Der Verfasser hat entsprechende Formen der accountability zum geltenden deutschen Recht sittenwidrigen Wettbewerbs und sittenwidriger Schädigung umfänglich untersucht und dabei festgestellt, dass es bereits eine recht umfängliche Tradition gesetzlicher Vorschriften und in der Rechtsprechung der gibt, die zwar die Vertragsfreiheit und sonstige Ausübunsformen der wettbewerblichen Handlungsfreiheit bejahen, aber zugleich Begrenzungen festlegen, in denen ein Konzept von Verantwortungsbereitschaft erkennbar ist. Nur Weniges ist hier hervorzuheben.

Für Kaufleute im Sinne der $\S \S 1 \mathrm{ff}$. HGB, die nach $\S \S 238$ ff. HGB die Rechnungslegungsvorschriften des Bilanzrechts zu befolgen haben, ist das Zusammenwirken strenger Ansatz- und Bewertungsregeln mit den sogenannten Wahlrechten relevant, da die dispositiven Bilanzmöglichkeiten stets auf objektiv nachvollziehbaren Abwägungsentscheidungen über kollidierende Interessen von shareholders (und stakeholders) beruhen müssen. Dazu hat man sich heute längst vom älteren Konzept einer Gewohnheitsethik im Sinne der sogenannten Grundsätze ordnungsmäßiger Bilanzierung verabschiedet und stattdessen auf wissenschaftlich anerkannte Methoden des risk management

77 Weiterführend zur Entstehung globaler Wirtschaftsordnung Teubner (2012), S. 120 ff., 211 ff.; neustens die Beiträge in Spießhofer (2017) und Hermann (2018). 
abgestellt (Engels 1962, S. 5; Stützel 1967, S. 319 f.; Herrmann 1977, S. 151, 167 ff.). Dem sollte die Rechtsprechung zum sogenannten Anstandsgefühl aller billig und gerecht Denkenden im Sinne der §§ 138, 826 BGB zumindest im kaufmännischen Bereich folgen.

Sehr viel weiter fortgeschritten ist in dieser Hinsicht das Aufsichtsrecht der MaRisk, ${ }^{78}$ das für das Risikomanagement von Banken und Versicherungen gilt. Besonderes Gewicht ist dabei auf die bilanziellen Wahlrechte und die sogenannten Eigenmodelle des Risikomanagements von Banken und Versicherungen zu legen, da deutlich wird, dass wettbewerbliche Handlungsfreiheit besteht, diese aber unter dem Vorbehalt steht, dass nach anerkannten Methoden des risk management eingehalten werden. Doch kann auch dies natürlich hier nur angedeutet werden (Hopt 2012).

Zum allgemeinen Vertragswirtschaftsrecht wird die Ethik der Risikozurechnung schon lange beachtet, soweit es um die Generalklauseln von Treu und Glauben und des Wegfalls der Geschäftsgrundlage gem. $\S \S 242$, 311, 313 BGB geht (Koller 1979; Köndgen 2000). In den beiden besonders risikorelevanten Gebieten des Bankund Versicherungsvertragsrechts hat sich inzwischen die Auferlegung von risikospezifischen Aufklärungsund Beratungspflichten gem. $\S 242$ in Verbindung mit $\S$ 491a BGB bzw. §§ 6, 61 VVG - wenngleich nur in engen Grenzen existenzgefährdender Geschäfte durchgesetzt (BGH NJW-RR 1988, 1512; ZIP 2008, 838). Doch besteht Erweiterungsbedarf (Metz 2012; Herrmann 2015/2016).

Zum Gesellschaftsrecht sind zunächst die Organisationsemphehlungen der Corporate Governance Kommission zu erwähnen, die für die börsennotierte AG mit § 161 eine - wenngleich bedingte - Legalität gewonnen haben. Steuerungsethik ist hier also geradezu primäre Normquelle, auch wenn die hier begründeten Ansätze weit über die herkömmlichen Lehren der Governance-Ethik von Wieland und Anderen hinausgehen (Wieland 20071; ders. 2007). ${ }^{79}$ Jedoch hat auch im Gesellschaftsrecht der BGH - trotz erheblicher Begründungsschwächen zum gewohnheitsethischen Beurteilungsansatz der $\S \S 138,826$ BGB - manche Einzelfälle durchaus im Sinne der hier vertretenen Erfordernisse der accountability entschieden hat. Das gilt v.a. für die berühmte Entscheidung im Fall Trihotel (BGHZ 173, 246), in der für die Durchgriffshaftung endlich

78 Rundschreiben 3/2009 (VA) - Aufsichtbehördliche Mindestanforderungen an das Risikomanagement (MARisk VA) www.BaFin. de/SharedDocs/Veroeffentlichungen/DE/Rundschreiben/rs_0903_ va_marisk.html (download 27.11.2014.); dazu Hopt (2012), S. 9, 23; ders. (2011), S. 15; Wymeersch (2010), S. 240; Mülbert (2010), S. $834,836 \mathrm{ff}$

79 Dazu z.T. krit. Oben zu I.1. zutreffend auf § 826 BGB abgestellt wurde (so schon Coing 1977). Der beherrschende Gesellschafter handelt sittenwidrig, wenn er die in Anspruch genommene Konzerngesellschaft dadurch finanziell ausplündert, dass er das haftende Vermögen auf andere Konzernmitglieder überträgt. Allerdings darf man nicht bei den Fallbesonderheiten des Risikotransfers durch bewusste Selbstausplünderung stehen bleiben, sondern muss schon immer dann sittenwidrige Schädigung bejahen, wenn Marktrisiken ohne nachvollziehbare Gründe der Risikokontrolle auf Marktpartner oder Dritte abgewälzt werden.

Des Weiteren hat im Gesellschaftsrecht der Gesetzgeber dafür gesorgt, das gewohnheitliche Sittlichkeitsdenken auf die Ebene der Verantwortungsethik zu bringen, indem er die Risikofinanzierung durch Gesellschafterdarlehen grundlegend verändert hat. Zunächst hat das Gesetz zur Modernisierung des $\mathrm{GmbH}-$ Rechts und zur Bekämpfung von Missbräuchen (MoMiG) von 2008 (BGBI. I, 2026) zwar zu einer gewissen De-Ethisierung i.S. von Anstandsbräuchen geführt, indem die zuvor geltende Rspr. über die Eigenkapitalzuführung "ordentlicher Kaufleute“ aufgegeben wurde (Conow 2015, S. 262, 276 f. u.ö.). Gesellschafterdarlehen in der Krise werden nicht mehr generell als unordentliche Kaufmannspraxis eingestuft, sondern bleiben ohne rechtsethische Beschränkung, wenn der Insolvenzeintritt letztlich erfolgreich vermieden wird oder so weit hinausgezögert werden kann, dass die Darlehensrückführung vor Insolvenzeintritt bereits mindestens $1 \mathrm{Jahr}$ zurückliegt (135 Abs. 1 Nr. 2 InsO).

Aber damit ist natürlich das Gros der Fälle nicht aus der strengen Gleichstellung mit Eigenkapital entlassen, in denen es schließlich doch vor Jahresablauf zur Insolvenz kommt. Hier soll es dann nicht einmal mehr, wie früher, darauf ankommen, ob die Gesellschaft unter Wettbewerbsbedingungen kein Darlehen bei der Hausbank oder einer anderen Bank mehr bekommen hätte. Vielmehr erfolgt die Rangrückstufung dann stets bloß deswegen, weil es ein Gesellschafter war, der das Darlehen gegeben hat. Das beruht ersichtlich auf Normzwecken wettbewerblicher Steuerungsanreize; und die Praxis hat tatsächlich sehr schnell Wege gefunden, über die Rangrückstufung über Darlehen stiller Gesellschafter mit Nebenabreden als sog. covenants zu vermeiden und dadurch wettbewerbliche Möglichkeiten der Bewältigung von Insolvenzrisiken mit angemessenem risk management zu nutzen (Heinrichs, 2009, S. 38; z.T. anders Servatius 2008, S. 102 ff.; Dette, 2012, S. 57 ff.). Wo dies nicht der Fall ist, hat die Literatur bereits, wenngleich bisher mit negativem Ergebnis, erwogen, die Rspr. zu § 826 BGB und der Trihotel-Fallgruppe zur Anwendung zu bringen (Conow, 
2015, S. 276 f.). Der Wertungszusammenhang ist aber unter den hier erörterten Aspekten der Verantwortungsbereitschaft offenkundig, so dass vorsichtige Analogien möglich erscheinen (Herrmann 2017, S. 28 ff.; ders. und Roth 2008/18 Band 2, Abschnitt IV.3d-g).

Zudem hat das BMF zu den steuerrechtlich hinzugehörenden Fragen des $\S 17$ Abs. 2 EStG wieder gegengesteuert, indem es die Fortsetzung der Anstandsmoral der älteren Rechtsprechung für die Beurteilung der sogenannten nachträglichen Anschaffungskosten durch die Finanzbehörde verfügt und besonders für die Sanierungsdarlehen von Gesellschaftern in der Krise der $\mathrm{GmbH}$ angeordnet hat (BMF $2010 \mathrm{zu}$ Nr. 4). Ob sich hier auf längere Sicht eine vermehrte Berücksichtigung hochethischer Aspekte der risikoethischen Steuerung durchsetzen lässt, muss derzeit allerdings offenbleiben. Der Absicht des BMF scheint es vorerst nicht zu entsprechen.

Speziell zum Kartellrecht ist wegen der besonderen Risikolagen bei Sanierungsfusionen hervorzuheben, dass v.a. die Berücksichtigung von Arbeitsplatzrisiken weitgehend anerkannt und mit besonderen Darlegungslasten des Risikomanagements verbunden worden ist. Die Rechtsprechung hat es hier schon seit den Zusammenschlusserlaubnissen in Thyssen/Hüller und Karstadt-Neckermann gebilligt, dass eine kartellbehördliche Genehmigung aus Gründen des Schutzes von Arbeitsplätzen erteilt wird. Will man dies nicht als

\section{Literatur}

Ahrens, Nils (2013), Unternehmensbeteiligungen und ihre Auswirkungen auf die Buchwertabfindung, Lohmar-Köln.

Albert, Hans und Wilhelm Meyer (2002), Grundlagen des ökonomischen Denkens, Tübingen.

Arendt, Hannah (1987), Vita Activa oder vom tätigen Leben, München - Zürich.

Arndt, Johann (1835), Sechs Bücher vom Wahren Christenthum, Reutlingen.

Assmann, Jan (2007), Das kulturelle Gedächtnis: Schrift, Erinnerung und politische Identität in frühen Hochkulturen, 6. Auflage, München.

Augustinus (2012), Gottesstaat, 5. Auflage, Einsiedeln.

Beck, Ulrich (2008), Der eigene Gott: Von der Friedensfähigkeit und dem Gewaltpotential der Religionen, Frankfurt am Main.

Biedermann, Reinhard (2007), Sozialstandards durch Private Governance: Zwei-Stufen-Kooperation in der globalen Spielzeugbranche, Reihe Internationale Politische Ökonomie, Band 5, Baden-Baden. allgemeine Zurücksetzung von Wettbewerbsinteressen hinter Sozialschutzbelange begreifen, so wird man vorauszusetzen haben, dass der Ast. besondere Risiken massenhafter Arbeitsmarktstörungen und Marktfriktionen nachweist (Herrmann 1984, S. 5 ff., 9 ff.). Das geht natürlich über Methoden des risk management hinaus, dürfte es aber auf Seiten der Antragsteller ausreichen lassen, dass die betriebswirtschaftlichen Angaben dazu gemacht werden. Die weitere Prüfung muss im Wege der Amtsermittlung von Seiten der Kartellbehörde erfolgen und könnte künftig mehr als bisher von Fragen der Missbrauchsgefahr wegen unverantwortlicher Risikoverfälschung bestimmt sein..

Damit wird der Beispielskatalog für Anwendungsfelder vertiefender Besinnung auf wettbewerbsethische Prinzipien abgeschlossen. Die Breite der Themen und Probleme macht hinreichend deutlich, dass es nicht länger ausreicht, anhand der Anstandsformel und gewohnheitlicher richterlicher Moralvorstellungen zu entscheiden, sondern dass es dringend meta-ethischer und historischer Analysen der Herkunft und Fortentwicklung von Maßstäben menschlichen Wirtschaftens im Wettbewerb bedarf. Vieles spricht dafür, den Marktteilnehmern eine Bereitschaft zur Verantwortung des von innen übernommenen Risikos abzuverlangen und sämtliche entgegengesetzte Maßnahmen dem Verdikt der Sittenwidrigkeit gem. §§ 138, 826 BGB zu unterwerfen, die verdeckte oder abgenötigte Risikotransfers enthalten.

Bienert, Walther und Christian Thomas (1934), Die Glaubenslehre des Christian Thomasius, Halle (Saale).

Boli, John (1995), The Economic Absorption of the Sacred, in: Robert Wuthnow (Hrsg.), Rethinking Materialism: Perspectives on the Spiritual Dimension of Economic Behavior, Grand Rapids, Michigan, S. 93-117.

Böhnke, Michael (2013), Wege zu Gott ohne Gott? In: Guido Bausenhart, Michael Böhnke und Dominik Lorenz (Hrsg.), Phänomenologie und Theologie im Gespräch: Impulse von Bernhard Weltle und Klaus Hemmerle, S. 451-473.

Bonhoeffer, Dietrich (1937), Nachfolge, 3. Auflage von Martin Kuske und Ilse Tödt (Hg.) (2002), Gütersloh.

Bundesministerium der Finanzen (2010), Auswirkungen des Gesetzes zur Modernisierung des $\mathrm{GmbH}$-Rechts und zur Bekämpfung von Missbräuchen (MoMiG) auf nachträgliche Anschaffungskosten gemäß § 17 Abs. 2 EStG; 
BMF-Schreiben vom 8. Juni 1999, Stellungnahme vom 21.10.2010, Bundessteuerblatt, 2010 I, S. $832 a ̈-834$.

Brecht, Martin (1981), Martin Luther, Band 1, Stuttgart.

Buck-Heeb, Petra und Andreas Dieckmann (2010), Selbstregulierung im Privatrecht, Tübingen.

Burckhard, Jakob (1860), Die Cultur der Renaissance in Italien: ein Versuch, Basel.

Coing, Helmut (1950/1993), Grundzüge der Rechtsphilosophie, 5. Auflage, Berlin - New York.

Coing, Helmut (1977), Zum Problem des sogenannten Durchgriffs bei juristischen Personen, Neue Juristische Wochenschrift 39, S. 1793-1797.

Conow, Andreas (2015), Vertragsbindung als Freiheitsvoraussetzung, Tübingen.

Darwin, Charles (1859), On the Origin of Species by Means of Natural Selection, or the Preservation of Favoured Races in the Struggle for Life, London.

Dette, Felix C. (2012), Verdeckte und atypische Beherrschungsverträge im Aktienrecht, BadenBaden.

Deuser, Hermann (2009), Religionsphilosophie, Berlin.

Di Fabio, Udo (2005), Die Kultur der Freiheit, München.

Diller, Hermann (2007), Preispolitik, 4. Auflage, Stuttgart.

Emmerich, Volker (2014), Kartellrecht, 13. Auflage, München.

Engels, Wolfram (1962), Betriebswirtschaftliche Entscheidungslehre im Licht der Entscheidungstheorie, Köln / Opladen.

Enskat, Rainer (2002), Metaphysik, in: Hans D. Betz, Don S. Browning, Bernd Janowski und Eberhard Jüngel (Hg.), Religion in Geschichte und Gegenwart, 4. Auflage, Band 5, S. 1171-1176.

Erasmus von Rotterdam (1975), Das Lob der Torheit, in: Werner Welzig (Hrsg.), Ausgewählte Schriften, Band 2, S. 1-211.

Enste, Dominik H. (2015), Markt und Moral: Eine ordnungsethische Reflexion, Positionen: Beiträge zur Ordnungspolitik aus dem Institut der deutschen Wirtschaft Köln, Nr. 69, Köln.

Fikentscher, Wolfgang (1997), Zwei Wertebenen, nicht zwei Reiche: Gedanken zu einer christlichsäkulären Wertontologie, in: Wolfgang Fikentscher, Steffen Heitmann, Josef Isensee, Martin Kriele, Nicolaus Lobkowicz und Rupert Scholz (Hrsg.), Wertewandel - Rechtswandel, Gräfeling, S. 121-166.

Finnis, John (1980 / 2011), Natural Law and Natural Rights, 2. Auflage, Oxford.
Franco, Giuseppe (2016), Von Salamanca nach Freiburg: Joseph Höffner und die Wirtschaftsethik der spanischen Spätscholastik, Journal for Markets and Ethics/Zeitschrift für Marktwirtschaft und Ethik 5, S. 423-432.

Frey, Bruno S. (1997), Markt und Motivation: Wie ökonomische Anreize die (Arbeits-) Moral verdrängen, München.

Fritsch, Michael (2011), Marktversagen und Wirtschaftspolitik, 8. Auflage, München.

Fritz, Carl-Thomas (2005), Die Transaktionskostentheorie und ihre Kritik sowie ihre Beziehung zum soziologischen Neo-Institutionalismus, Bern u.a.

Goethe, Johann Wolfgang von und Friedrich Schiller (1797), Xenien, in: Friedrich Schiller (Hrsg.), Musen-Almanach für das Jahr 1797, Tübingen.

Graf, Hans-Georg (1978), Muster-Voraussagen und Erklärungen des Prinzips bei F. A. von Hayek, Tübingen.

Groß, Steffen W. (2010), Warum sich Ökonomen (wieder) mehr mit Philosophie beschäftigen sollten - und Philosophen (wieder) mit Ökonomie, ORDO - Jahrbuch für die Ordnung von Wirtschaft und Gesellschaft 61, S. 75-93.

Groß, Walter und andere Autoren (2001), creatio ex nihilo, in: Hans D. Betz, Don S. Browning, Bernd Janowski und Eberhard Jüngel (Hg.), Religion in Geschichte und Gegenwart, 4. Auflage, Band 2, S. $486-487$.

Habermas, Jürgen (1962/1975), Strukturwandel der Öffentlichkeit: Untersuchungen zur einer Kategorie der bürgerlichen Gesellschaft, 7. Auflage, Neuwied u.a.

Hailer, Martin (2014), Religionsphilosophie, Göttingen - Bristol.

Hansen, Reimer (2006), Glaubensfreiheit und Toleranz im konfessionellen Zeitalter, in: Matthias Mahlmann und Hubert Rottleuthner (Hrsg.), Ein neuer Kampf der Religionen? Staat, Recht und religiöse Toleranz, Berlin, S. 43-74.

Hattenhauer, Hans (1969), Hermann Conring und die deutsche Rechtsgeschichte, Schleswig-Holsteinische Anzeigen 1969, S. 69-76.

Hayek, Friedrich August von (1967), Grundsätze einer liberalen Gesellschaftsordnung, ORDO - Jahrbuch für die Ordnung von Wirtschaft und Gesellschaft 18 , S. 11-33.

Heinrichs, Stefan (2009), Covenants als Alternative zum institutionellen Gläubigerschutz: Eine rechtsvergleichende und ökonomische Analyse, Berlin.

Herdzina, Klaus (1999), Wettbewerbspolitik, 5. Auflage, Stuttgart. 
Herrmann, Harald (1971), Das Verhältnis von Recht und pietistischer Theologie bei Christian Thomasius, Kiel.

Herrmann, Harald (1977), Organisations- und Entscheidungslehre im Unternehmensverfassungsrecht, in: Norbert Horn und Reinhard Tietz (Hrsg.), Sozialwissenschaften im Studium des Rechts, Band 1, Zivil- und Wirtschaftsrecht, München, S. 151-172.

Herrmann, Harald (1979), Verbandskonzentration und Großunternehmen: Zur Bedeutung des Vereinsrechts für die Organisation von Großunternehmen in den Interessenverbänden der deutschen und englischen Industrie (1871-1914), in: Norbert Horn und Jürgen Kocka, (Hg.), Recht und Entwicklung der Großunternehmen im 19. und 20. Jahrhundert: Wirtschafts-, sozial- und rechtshistorische Untersuchungen zur Industrialisierung in Deutschland, Frankreich, England und den USA, Göttingen, S. 647-676.

Herrmann, Harald (1984), Interessenverbände und Wettbewerbsrecht: Ein deutsch-amerikanischer Vergleich zum Recht der unberechtigten Verfahrenseinleitung, Selbstbeschränkungsabkommen und Wettbewerbsregeln, Baden-Baden.

Herrmann, Harald (2006), Antitrust Law Compliance and Professional Governance: How can the European Commission Trigger Competitive Self-Regulation in: Claus Dieter Ehlermann und Isabela Atanasiu, European Competition Law Annual 2004: The Relationship between Competition Law and the (Liberal) Professions, Oxford / Portland Oregon, S. 101-130.

Herrmann, Harald (2005), Das wertphilosophische Leitbild persönlicher Verantwortlichkeit und Toleranz im internationalen Kartellrecht, in: Harald Herrmann und Kai-Ingo Voigt (Hg.), Globalisierung und Ethik, Heidelberg, S. 63-75.

Herrmann, Harald (2009), Wertewechsel in einer Rechtsanwaltsethik globalen Diskurses, Anwaltsblatt 12/2009, Bonn, S. 812-820.

Herrmann, Harald (2006), Zillmerungsregeln in der Lebensversicherung und kein Ende, Versicherungsrecht - Zeitschrift für Versicherungsrecht, Haftungs- und Schadensrecht, Jg. 57, Karlsruhe S. 7-14.

Herrmann, Harald (2006), Grundlehren BGB / HGB, Band 1, Nürnberg.

Herrmann, Harald (2015), Risikomanangement in der Kreditversicherung: Bloßes Werbeversprechen oder verbindliche Zusage in Krisenzeiten? In: Versicherungsrecht - Zeitschrift für Versicherungsrecht, Haftungs- und Schadensrecht, Jg. 66, Karlsruhe, 275-292.

Herrmann, Harald (2016), Risikofrüherkennungspflichten und kundengerechte Kreditberatung: Zur Genese des Prinzips geteilten Risikovertrauens. Zeitschrift für Bankrecht und Bankwirtschaft 2016 (4), S. 260-271.

Herrmann, Harald, Fünfzehn Jahre Neues Wirtschaftsrecht, in Sonderheft NWiR 2017, www.NWiR.de

Herrmann, Harald (2018, im Erscheinen), Rechtsethik im Kulturraum des Westens.

Herrmann, Harald und Stefan Roth (2008/18), Gesellschafts- und Konzernrecht für Wirtschaftsjuristen: Grundlehren der Governance - Unternehmenspublizität - Verbandsdemokratie, Berlin.

Herms, Eilert (2001), Kontingenz, in: Hans D. Betz, Don S. Browning, Bernd Janowski und Eberhard Jüngel (Hg.), Religion in Geschichte und Gegenwart, 4. Auflage, Band 4, S. 1647-150.

Hobbes, Thomas (1651), Leviathan oder Stoff, Form und Gewalt eines kirchlichen und bürgerlichen Staates, Neuauflage von Iring Fetscher (Hg.) (1984) Frankfurt am Main.

Hobbes, Thomas (1651), Leviathan: Revised Student Edition, Cambridge Texts in the History of Political Thought, Neuauflage von Richard Tuck (Hg.) (1991), Cambridge.

Höffe, Otfried (1981), Widersprüche im Leviathan: Zum Gelingen und Versagen der Hobbesschen Staatsbegründung, in: Otfried Höffe (Hg.): Thomas Hobbes: Anthropologie und Staatsphilosophie, Freiburg, S. 113-121.

Höffner, Joseph (2014), Wirtschaftsethik und Monopole im fünfzehnten und sechzehnten Jahrhundert, in: Jörg Althammer und Ursula Nothelle-Wildfeuer, Joseph Höffner - Ausgewählte Schriften, Band 3, Paderborn, S. 33-78.

Holthaus, Stephan (2006), Selbsterfahrung und Selbstbestimmung, Vortragsfassung; URL: http:// www.ethikinstitut.de/fileadmin/ethikinstitut/ .../individualismus_APS.pdf (abgerufen am 14.09.2016).

Homann, Karl (1999), Die Relevanz der Ökonomik für die Implementation ethischer Zielsetzungen, in: Wilhelm Korff u.a. (Hrsg.), Handbuch der Wirtschaftsethik, Gütersloh, S. 322-343.

Homann, Karl und Andreas Suchanek (2005), Ökonomik. Eine Einführung, 2. Auflage, Tübingen.

Homann, Karl und Corinna Gruber (2014), Die Marktwirtschaft und ihre intellektuellen Kritiker, Diskussionspapier des Roman-Herzog-Instituts, München.

Hommelhoff, Peter und Detlef Kleindiek (1992), Flexible Finanzierungsinstrumente im $\mathrm{GmbH}$-Recht, in: Marcus Lutter u.a. (Hrsg.), Festschrift 100 Jahre $\mathrm{GmbH}$, Köln, S. 421-445. 
Hoppmann, Erich und Ernst-Joachim Mestmäcker (1974), Normzwecke und Systemfunktionen im Recht der Wettbewerbsbeschränkungen, Tübingen.

Horsch, Andreas und Tanja Rathmann (2012), Kreditrisikotransfer durch Kreditversicherung, Hamburg.

Hopt, Klaus (2011), Comparative Corporate Governance, American Journal of Comparative Law 1 (2011), S. 1-23.

Hopt, Klaus und Gottfried Wohlmannsstetter (Hrsg., 2012), Handbuch Corporate Governance Banken, München.

Hopt, Klaus und Gunther Teubner (Hrsg., 1985), Corporate Governance and Directors' Liabilities, Berlin - New York.

Horn, Norbert (1979), Aktienrechtliche Unternehmensorganisation in der Hochindustrialisierung (1860-1920), in: Norbert Horn und Jürgen Kocka (Hrsg.), Recht und Entwicklung der Großunternehmen im 19. und frühen 20. Jahrhundert, Göttingen, S. 123-182.

Horn, Norbert (1995), Einleitung vor $§ 1$ HGB, in: Heymann (Hrsg.) Handelsgesetzbuch, 2. Auflage, Berlin - New York, S. 1-78.

Horn, Norbert (2007), Utilitarismus im aufgeklärten Naturrecht von Thomasius und Wolff, in HansPeter Haferkamp und Tilman Repgen (Hrsg.), Usus Modernus Pandectarum, Köln u.a., S. 45-62.

Horn, Norbert (2016), Einführung in die Rechtswissenschaft und Rechtsphilosophie, 6. Auflage, Heidelberg.

Huber, Wolfgang (2012), Von der Freiheit, München.

Hume, David (2005), An Enquiry concerning the Principles of Morals, herausgegeben von Tom Beauchamp, Radford/VA.

Immenga, Ulrich (1976), Politische Instrumentierung des Kartellrechts? In: Recht und Staat in Geschichte und Gegenwart, Heft 461.

James, William (1994), Was ist Pragmatismus, 2. Auflage, Hamburg.

Jann, Werner und Kai Wegrich (2004), in: Arthur Benz und Nicolai Dose (Hrsg.), Governance - Regieren in komplexen Systemen, 2. Auflage, Wiesbaden, S. $198-214$.

Joffe, Josef (2017), Bitte genau hierhin, in: Die Zeit vom 12. Oktober 2017, S. 30.

Kant, Immanuel (1793), Über dem Gemeinspruch: Das mag in der Theorie richtig sein, taugt aber nicht für die Praxis, in: Immanuel Kant, Gesammelte Schriften, Akademieausgabe ab 1902, herausgegeben von Wilhelm Weischedel, Darmstadt 1968.
Kant, Immanuel (1788), Kritik der praktischen Vernunft, 2. Auflage, herausgegeben von Wilhelm Weischedel, Darmstadt 1968.

Kant, Immanuel (1787), Kritik der reinen Vernunft, 2. Auflage, herausgegeben von Wilhelm Weischedel, Darmstadt 1968.

Kant, Immanuel (1785), Metaphysik der Sitten, 1785, 2. Auflage 1786, herausgegeben von Wilhelm Weischedel, Darmstadt 1968.

Kaltenborn-von Stachau, Karl (1848), Die Vorläufer des Hugo Grotius auf dem Gebiete des lus Naturae et Gentium sowie der Politik im Reformationszeitalter, Leipzig.

Kaufmann, Arthur (1991), Die Naturrechtsrenaissance der ersten Nachkriegsjahre, in: Michael Stolleis (Hrsg.), Die Bedeutung der Wörter. Studien zur europäischen Rechtsgeschichte. Festschrift für Sten Gagnér zum 70. Geburtstag, München, S. 105-132.

Kerber, Wolfgang (1999), Besprechung von Herdzina, in: Wirtschaft und Wettbewerb 49, S. 1203-1204.

Keßler, Jürgen und Hans-W. Micklitz (2007), Die Richtlinie 2005/29/EG über unlautere Geschäftspraktiken im binnenmarktinternen Geschäftsverkehr zwischen Unternehmen und Verbrauchern, in: Betriebs-Berater Spezial 13/2007, S. 1-23.

Kieninger, Eva-Maria (2002), Wettbewerb der Privatrechtsordnungen im europäischen Binnenmarkt, Tübingen.

Köndgen, Johannes (2000), Entwicklungen des Bankkreditrechts in den Jahren 1995-1999, in: Neue Juristische Wochenschrift, S. 468-470.

Koller, Ingo (1979), Die Risikozurechnung bei Vertragsstörungen in Austauschverträgen, München.

Koller, Ingo (2006), Privatisierung des Rechts, in: Archiv für die civilistische Praxis 206, S. 477-525.

Koppensteiner, Hans Georg (2007), Marktbezogene Unlauterkeit und Missbrauch von Marktmacht, in: Wettbewerb in Recht und Praxis, S. 475-489.

Koslowski, Peter (1982), Ethik des Kapitalismus, Tübingen.

Küng, Hans (1990), Projekt Weltethos, München.

Küng, Hans (2006), Das Christentum, 5. Auflage, München.

Küng, Hans (2008), Leitlinien zum Weltdenken, in: HansMartin Schönherr-Mann (Hrsg.), Miteinander leben lernen, München - Zürich, S. 344-354.

Kuhn, Thomas (1996), Die Struktur wissenschaftlicher Revolutionen, Frankfurt.

Kuntz, Thilo (2016), Gestaltung von Kapitalgesellschaften zwischen Freiheit und Zwang, Tübingen. 
Lachmann, Werner (1989), Wirtschaft und Ethik, 2. Auflage, Neuhausen - Stuttgart.

Lachmann, Werner (2013), Religion, Wirtschaft, Wirtschaftsethik, in: Journal for Markets and Ethics/Zeitschrift für Marktwirtschaft und Ethik 1, S. 3-29.

Linke, Detlef (2005), Religion als Risiko, 2. Auflage, Hamburg.

Locke, John (1704), Two Treatises of Government. The Works, 1704, 11. Auflage 1812, Nachdruck 1963, London.

Luce, Edward (2017), The Retreat of Western Liberalism, London.

Luig, Klaus (1998), Römisches Recht, Naturrecht und nationales Recht, Goldbach.

Luther, Martin (2000-2007), Martin Luthers Werke, Kritische Gesamtausgabe, Stuttgart.

Luther, Martin (2014), An den christlichen Adel deutscher Nation von des christlichen Standes Besserung, bearbeitet und kommentiert von Thomas Kaufmann, Kommentare zu Schriften Luthers, Band 3, Tübingen.

Luther, Martin (2007), Von der Freiheit eines Christenmenschen, bearbeitet und kommentiert von Reinhold Rieger, Kommentare zu Schriften Luthers, Band 1, Tübingen.

Marx, Karl (1968), Das Kapital, Erster Band, Berlin.

Mattern, Isabel (2011), Das Informationsmodell im VVG unter Berücksichtigung der Auswirkungen auf die AGB-Kontrolle, Karlsruhe.

McConica, James (1991), Erasmus, Oxford.

Meinecke, Friedrich (1946), Die Entstehung des Historismus, 2. Auflage, München - Berlin.

Metz, Rainer (2012), Erläuterungspflichten bei Verbraucherkreditverträgen, in: Neue Juristische Wochenschrift 2012, S. 1990-1995.

Meyer, Wilhelm (2002), Grundlagen ökonomischen Denkens, Tübingen.

Meyer, Wilhelm (2014), Besserung der Welt durch Ethisierung der Ökonomik? ORDO - Jahrbuch für die Ordnung von Wirtschaft und Gesellschaft 65, S. 387-411.

Melanchthon Philip (1977), Melanchthons Briefwechsel, herausgegeben von Heinz Scheible, Tübingen.

Mestmäcker, Ernst-Joachim (1984), Der verwaltete Wettbewerb, Tübingen.

Mestmäcker, Ernst-Joachim (1994), Aufklärung durch Recht, in: Hans Friedrich Fulda und Horst-Peter Horstmann (Hrsg.), Vernunftbegriffe in der Moderne, Stuttgart, S. 55-72.

Mestmäcker, Ernst-Joachim (2010), Der gestrandete Leviathan. Über Gedanken- und Religionsfrei- heit in der bürgerlichen Gesellschaft, in: Festschrift für Dieter Reuter zum 70. Geburtstag, S. 1293-1302.

Möllers, Thomas M. J. (Hrsg., 2008), Standardisierung durch Markt und Recht, Baden-Baden.

Möllers, Thomas M. J. (2008), Compliance-Gesetz und Compliance-Kodex, in: Betriebs-Berater, Nr. 25, Die erste Seite.

Möschel, Wernhard (1974), Der Oligopolmissbrauch im Recht der Wettbewerbsbeschränkungen, Tübingen.

Montenbruck, Axel (2013), Mittelwelt und Drei-MittelMensch, 2. Auflage, Berlin.

Montenbruck, Axel (2013), Demokratischer PräambelHumanismus, 4. Auflage, Berlin.

Mounk, Yasha (2017), The Age of Responsibility, Cambridge/MA.

Müller-Armack, Alfred (1970), Der Moralist und der Ökonom, ORDO - Jahrbuch für die Ordnung von Wirtschaft und Gesellschaft 21, S. 19-41.

Mülbert, Peter, Finanzmarktregulierung, in: Juristenzeitung, S. 834-438.

Nida-Rümelin, Julian (2011), Die Optimierungsfalle, München.

Nida-Rümelin, Julian (1997), Praktische Kohärenz, in: Zeitschrift für philosophische Forschung, S. 175-192.

Nitsche, Stefan A. (2002), König David, Gütersloh.

Pfordten, Dietmar von der (2011), Rechtsethik, 2. Auflage, München.

Precht, Richard D. (2013/17), Erkenne die Welt, 6. Auflage, München; Bd.2: Erkenne dich selbst.

Rawls, John (1971), A Theory of Justice, Cambridge.

Rentsch, Thomas (2000), Negativität und praktische Vernunft, Frankfurt am Main.

Rentsch, Thomas (2005), Gott, Berlin u.a.

Reuter, Dieter (1973), Privatrechtliche Schranken der Perpetuierung von Unternehmen, Frankfurt am Main.

Rieger, Reinhold (1999), Art. Duns Scotus, Johannes, in: Religion in Geschichte und Gegenwart, Band 2, Sp. 1016-1019.

Ritschl, Albrecht (1884), Geschichte des Pietismus, Band 2, Bonn.

Röpke, Wilhelm (1979), Jenseits von Angebot und Nachfrage, Bern - Stuttgart.

de Roover, Raymond (1955), Scholastic Economics, in: Quarterly Journal of Economics 69, S. 161-179.

Rüping, Heinrich (1968), Die Naturrechtslehre des Christian Thomasius und ihre Fortbildung in der Thomasius-Schule, Bonn.

Russell, Bertrand (20013), Human Knowledge: Its Scope and Limits, London - New York. 
Sandel, Michael (2012), What Money Can't Buy, New York.

Schatz, Klaus (1990), Der päpstliche Primat, Würzburg.

Scheible, Heinz (1997), Melanchthon: Eine Biographie, München.

Scheible, Heinz (2010), Aufsätze zu Melanchthon, Tübingen.

Schilling, Heinz (2012), Martin Luther, München.

Schilling, Heinz (2009), Das konfessionelle Europa, in Hans G. Kippenberg und Kocku von Stuckrad (Hrsg.), Europäische Religionsgeschichte: Ein mehrfacher Pluralismus. Göttingen, 289-338.

Schmidt, Helmut (2009), Der Markt ist keine sichere Bank, in: Die Zeit vom 4. Mai 2009.

Schmidt, Ingo (1999), Wettbewerbspolitik und KartelIrecht, 6. Auflage, München.

Schmidt, Karsten (2006), Satzungsfreiheit, Individualrechte und Bindung von Rechtsnachfolgern, in: Sibylle Kessler u.a. (Hrsg.), Formale Freiheitsethik und materiale Verantwortungsethik, München, S. 9-32.

Schmitz, Wolfgang (Hrsg., 1982), Was macht den Markt sozial? Wien.

Schneider-Flume, G., Grundkurs Dogmatik, 2. Auflage, Göttingen.

Schneiders, Werner (1971), Naturrecht und Liebesethik. Zur Geschichte der praktischen Philosophie mit Hinblick auf Christian Thomasius. Hildesheim New York 1971.

Schröder, Jan (2001), Recht als Wissenschaft. Geschichte der juristischen Methode vom Humanismus bis zur historischen Schule, München.

Schelika, Arnulf (2007), Kontingenz und Vorsehung im Werk Immanuel Kants, in: Udo Kern (Hrsg.): Was ist und was soll sein, Natur und Freiheit bei Immanuel Kant, Berlin, S. 373-478.

Schubert, Hans-Joachim, Hans Joas und Harald Wenzel (2010), Pragmatismus zur Einführung, Hamburg.

Schumpeter, Joseph (1954), History of Economic Analysis, New York.

Schwöbel, Christoph (2000), Art. Gott, in: Religion in Geschichte und Gegenwart, Band 3, Sp. 1116.

Sedlacek, Tomas (2013), Die Ökonomie von Gut und Böse, München.

Seeberg, Erich (1920), Christian Thomasius und Gottfried Arnold, in: Neue kirchliche Zeitschrift 31, S. 337-358.

Seibert, Ulrich (2010), Deutschland im Herbst - Erinnerungen an die Entstehung des Finanzmarktstabilisierungsgesetzes im Oktober 2008, in: Stefan Grundmann u.a. (Hrsg.), Unternehmen, Markt und Verantwort. Festschrift für Klaus J. Hopt zum 70. Geburtstag, Band 2, Berlin - New York, S. 2525-2547.

Sen, Amartya (2011), Ökonomie für den Menschen, 5. Auflage, München.

Shiller, Robert (2012), Märkte für Menschen, Frankfurt am Main - New York.

Smith, Adam (1980), Essays on Philosophical Subjects, in: D. Daiches Raphael und Andrew Skinner (Hrsg.), The Glasgow Edition of the Works and Correspondence of Adam Smith, Oxford.

Smith, Adam (1994), Die Theorie der ethischen Gefühle, Hamburg.

Spießhofer, Birgit (Hrsg.), Unternehmerische Verantwortung. Zur Entstehung einer globalen Wirtschaftsordnung, Baden-Baden.

Stegmaier, Werner (2008), Philosophie der Orientierung, Berlin.

Stock, Konrad (2011), Einleitung in die systematische Theologie, Berlin.

Strawson, Galen (1994), The Impossibility of Moral Responsibility, in: Philosophical Studies 74, S. 5-24.

Strawson, Galen (1986), Freedom and Belief, Oxford.

Stützel, Wolfgang (1967), Bemerkungen zur Bilanztheorie, in: Zeitschrift für Betriebswirtschaft 37, S. 314-331.

Suchanek Andreas und Nick Lin-Hi (2006), Eine Konzeption wirtschaftlicher Verantwortung, Wittenberg-Zentrum für globale Ethik, Diskussionspapier 2006-7.

Stigler, George J., Die vollständige Konkurrenz im historischen Rückblick, in Klaus Herdzina (Hrsg.) Wettbewerbstheorie, Frankfurt am Main, S. 30-51.

Sturm, Roland (2009), Politik in Großbritannien, Wiesbaden.

Teubner, Gunther (1978), Organisationsdemokratie und Verbandsverfassung, Tübingen.

Teubner, Gunther (2012), Verfassungsfragmente, Berlin.

Thaler, Richard und Cass R. Sunstein (2008), Nudge, Improving decisions about health, wealth, and happiness, New Haven.

Thomas von Aquin (1977), Summa Theologica, deutschlateinsche Ausgabe, herausgegeben von der Philosophisch-Theologischen Hochschule Walberberg.

Thomasius, Christian (1720/1963), Institutionum Jurisprudentiae Divinae, Buch I und III, 7. Auflage, Halle, Neudruck 1963.

Thomasius, Christian (1696/1968), Ausübung der Sittenlehre, Halle, Neudruck 1968.

Thomasius, Christian (1960), Über die Folter, herausgegeben von Rolf Lieberwirth, Weimar. 
Todt, Horst, Freiheit und Utilitarismus, in Ott/Schäfer (Hrsg.), Ökonom. Probleme des Zivilrechts, 1991 S. 1-17.

Troeltsch, Ernst (1913), Die Bedeutung des Begriffs der Kontingenz, in: Gesammelte Schriften, Band 2, S. 769-792.

Utzschneider, Helmut (2005), Art. Traditionskritik/ Traditionsgeschichte der Bibel, in: Religion in Geschichte und Gegenwart, Band 8, 2005, Sp. 528-529.

Vogl, Joseph (2011), Das Gespenst des Kapitalismus, Zürich.

Weber, Max (1985), Wirtschaft und Gesellschaft, 6. Auflage, Tübingen.

Weber, Max (1998), Die protestantische Ethik und der Geist des Kapitalismus, in: Max Weber, Gesammelte Aufsätze zur Religionssoziologie, 9. Auflage, Band 1.

Welzel, Hans, Naturrecht und materiale Gerechtigkeit, 4. Auflage, Göttingen.

Wieacker, Franz (1967), Privatrechtsgeschichte der Neuzeit, 2. Auflage, Göttingen.
Whitehead, Alfred North (1985), Wie entsteht Religion? Frankfurt am Main.

Wieland, Josef (2007), Die Ethik der Governance, 5. Auflage, Marburg.

Wieland, Josef (2001), Eine Theorie der Governanceethik, in: Zeitschrift für Wirtschafts - und Untenehmensethik 2, S. 8-33.

Winkler, Gerhard B. (1999), Art. Erasmus, in: Religion in Geschichte und Gegenwart, Band 2, Sp. 1380-1384.

Worsdörfer, Manuel und Carsten Dethlefs (2012), Homo oeconomicus oder Homo culturalis? In: ORDO - Jahrbuch für die Ordnung von Wirtschaft und Gesellschaft 63, S. 135-157.

Würthwein, Ernst (1974), Die Erzählung von der Thronfolge Davids, Zürich.

Wymeersch, Eddy (2010), The institutional reforms of the European Financial Supervisory System, Ghent Univ. Financial Law Institute Working Paper No. 2010-01.

Zaczyk, Rainer (2014), Selbstsein und Recht, Frankfurt am Main. 
